\title{
Vie et mort d'un support d'outil : Chaînes opératoires de réaménagement des pièces façonnées unifacialement du technocomplexe Itaparica (Brésil Central)
}

\author{
Antoine Lourdeau ${ }^{1,2}$ \\ 1. Muséum National d'Histoire Naturelle, Département de Préhistoire, Musée de l'Homme, 17 place du \\ Trocadéro, 75116 Paris, France. \\ 2. Universidade Federal de Sergipe, Programa de Pós-graduação em Arqueologia, Laranjeiras, Brésil. \\ Email: antoine.lourdeau@mnhn.fr
}

\section{Résumé :}

Le technocomplexe Itaparica comprend la plupart des productions lithiques de la transition Pléistocène-Holocène et de l'Holocène ancien dans le centre du Brésil. Il se caractérise par des industries basées sur une complémentarité entre des pièces façonnées unifacialement et des éclats retouchés. Les pièces façonnées unifacialement sont historiquement les vestiges les plus emblématiques de ce technocomplexe. Elles correspondent à un support pouvant contenir plusieurs outils. De nombreux indices démontrent que ces pièces avaient une longue durée de vie utile et passaient par différents états techniques successifs. A partir de la riche collection de l'abri GO-JA-01 (État de Goiás, Brésil), nous présentons ici une étude des chaînes opératoires de réamenagement des pièces façonnées unifacialement du technocomplexe Itaparica. Par les différents indices de raffûtage et de réaménagement, il est possible de définir deux schémas opératoires d'exploitation de ces outils au cours du temps, jusqu'à leur dénaturation. En revanche, bien que les réaménagements successifs aient un impact sur la forme et le volume des supports, ils ne peuvent pas expliquer toute la variabilité de ces artefacts. La structure des supports semble se maintenir au cours de la vie technique de ceux-ci. Il y a donc une diversité des intentions dès le début de la production des pièces façonnées unifacialement du technocomplexe Itaparica.

Mots-clés : chaîne opératoire; technologie lithique; vie utile d'un outil; pieces façonnées unifacialement; technocomplexe Itaparica; préhistoire du Brésil

\section{Introduction}

Une cinquantaine d'années après sa formulation (Leroi-Gourhan 1964), la notion de chaîne opératoire demeure un outil extrêmement efficace pour l'étude de la production des outils de pierre. Elle l'est tout autant pour l'approche des différents états techniques de ces outils au cours de leur utilisation. Nous en donnons ici un exemple avec le cas des pièces façonnées unifacialement du technocomplexe Itaparica.

Published by the School of History, Classics and Archaeology, University of Edinburgh ISSN: 2055-0472. URL: http://journals.ed.ac.uk/lithicstudies/

This work is licensed under a Creative Commons Attribution 2.5 UK: Scotland License. 
Le technocomplexe Itaparica est un ensemble techno-culturel cohérent dans un vaste espace qui couvre tout le Brésil Central durant la transition Pléistocène-Holocène et l'Holocène ancien (Figure 1). Il correspond au premier moment de peuplement relativement dense de cette région par des groupes de chasseurs-cueilleurs non spécialisés (Bueno et al. 2013; Lourdeau 2015). La caractérisation de son système technique ne se limite pas à la présence de ces pièces façonnées unifacialement (aussi appelées lesmas, "limaces” en portugais, ou encore outils plano-convexes ou unifaces). L'industrie du technocomplexe Itaparica se base sur une complémentarité fonctionnelle entre ces pièces, présentes dans les ensembles lithiques dans des proportions variables, des outils sur éclats retouchés assez normalisés et, de façon tout-à-fait ponctuelle, des pointes de projectile bifaciales (Schmitz 1987a; Fogaça 1995; Lourdeau 2010).

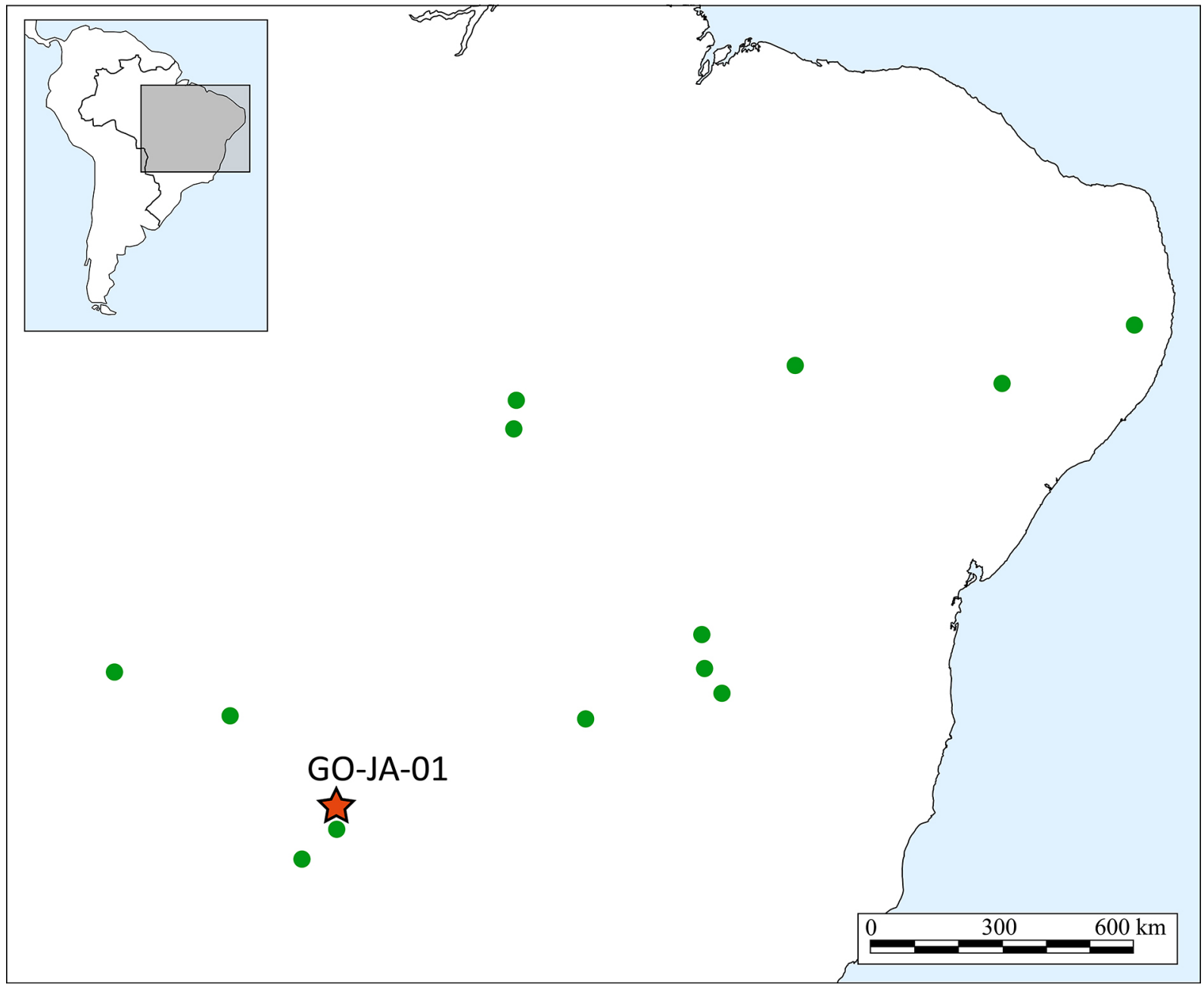

Figure 1. Carte des principaux sites du technocomplexe Itaparica et localisation de GO-JA-01.

Figure 1. Map of main sites of the Itaparica technocomplex and location of GO-JA-01 rockshelter.

\subsection{Les pièces façonnées unifacialement du technocomplexe Itaparica}

Les études techno-fonctionnelles effectuées sur les pièces façonnées unifacialement du technocomplexe Itaparica ont montré qu'elles constituent un support original (Lourdeau 2010; 2014) (Figure 2).

L'analyse techno-fonctionnelle permet, à partir de la lecture diacritique des stigmates de taille, une compréhension globale des industries lithiques par l'approche intégrée des modes de production et des objectifs de taille (les outils produits) (Boëda 1997; 2001; Soriano 2000). Ces outils sont caractérisés par leurs propriétés structurelles et leur potentiel fonctionnel. Au 
contraire de l'analyse tracéologique qui accède à la fonction et au fonctionnement des outils par les traces d'utilisation, l'analyse techno-fonctionnelle aborde la sphère fonctionnelle par le biais des intentions du tailleur lors de la production de l'outil. Les deux méthodes sont donc complémentaires.

Selon cette approche, les pièces façonnées unifacialement du technocomplexte Itaparica correspondent à un même concept de taille, qui repose sur :

- un principe volumétrique : ces artefacts ont un volume allongé assez épais, symétrique selon l'axe longitudinal et dont l'une des faces est plane.

- un principe productionnel : ce support est obtenu à partir d'un éclat de grande dimension, dont la face supérieure a ensuite été façonnée. Les enlèvements de façonnage sont plus ou moins envahissants selon les cas. Ils sont détachés à partir du bord du support selon une direction centripète et s'étendent sur tout le pourtour ou presque. La face inférieure de l'éclat-support constitue toujours la face plane de la structure volumétrique.

- un principe fonctionnel: ce volume supporte au moins un outil, dont la partie transformative est située à l'extrémité apicale et est symétrique selon l'axe longitudinal. La préhension de cet outil se fait dans la moitié opposée à la partie transformative. L'emmanchement de ces pièces est probable dans plusieurs cas.

Au-delà de ces caractères communs, il existe une variabilité manifeste de ces artefacts. Deux catégories volumétriques se différencient nettement. On distingue d'une part un ensemble de pièces épaisses à profil asymétrique (dont l'épaisseur minimale se situe au niveau de l'extrémité contenant la partie transformative) ou symétrique (Figure 2, n³ et 5), et d'autre part un ensemble de pièces peu épaisses à profil symétrique, dont l'épaisseur est constante tout le long de l'outil (Figure 2, ${ }^{\circ} 1,2$, 4 et 6). Par ailleurs les sections transversales sont semi-circulaires, trapézoïdales ou triangulaires.

A cette variabilité volumétrique s'ajoute une variabilité des propriétés technofonctionnelles. Les extrémités transformatives possèdent des caractères de délinéation, d'angle, de surface relativement variés d'une pièce à l'autre. Il en découle une évidente variété des potentiels fonctionnels de ces outils. Il existe aussi différentes structures fonctionnelles. Certains de ces artefacts sont conçus pour ne contenir qu'un seul outil, dont la partie transformative est toujours située à l'extrémité de la pièce ("pièces outil”) (Figure 2, $\mathrm{n}^{\circ} 1,3$ et 5). D’autres artefacts, en revanche, sont produits de façon à constituer des matrices au sein desquelles plusieurs outils peuvent être confectionnés et utilisés de manière contemporaine ou l'un après l'autre (“pièces support-d'outils”) (Boëda 1997; 2001) (Figure 2, $n^{\circ} 2$, 4 et 6). Les parties transformatives de ces outils, outre celle qui se trouve systématiquement à une extrémité, sont situées sur les côtés ou à l'autre extrémité (Lourdeau 2010).

Un trait marquant des pièces façonnées unifacialement du technocomplexe Itaparica est leur longue durée de vie utile. Le support offre une réserve de matière et sa structure planoconvexe permet de nombreuses phases de raffûtage et de réaménagement avant l'exhaustion de la pièce. C’est cet aspect qui est développé ici. Par une analyse des différents stigmates et indices de reprise de ces supports, nous proposons une restitution des chaînes opératoires de leur gestion au cours du temps. 

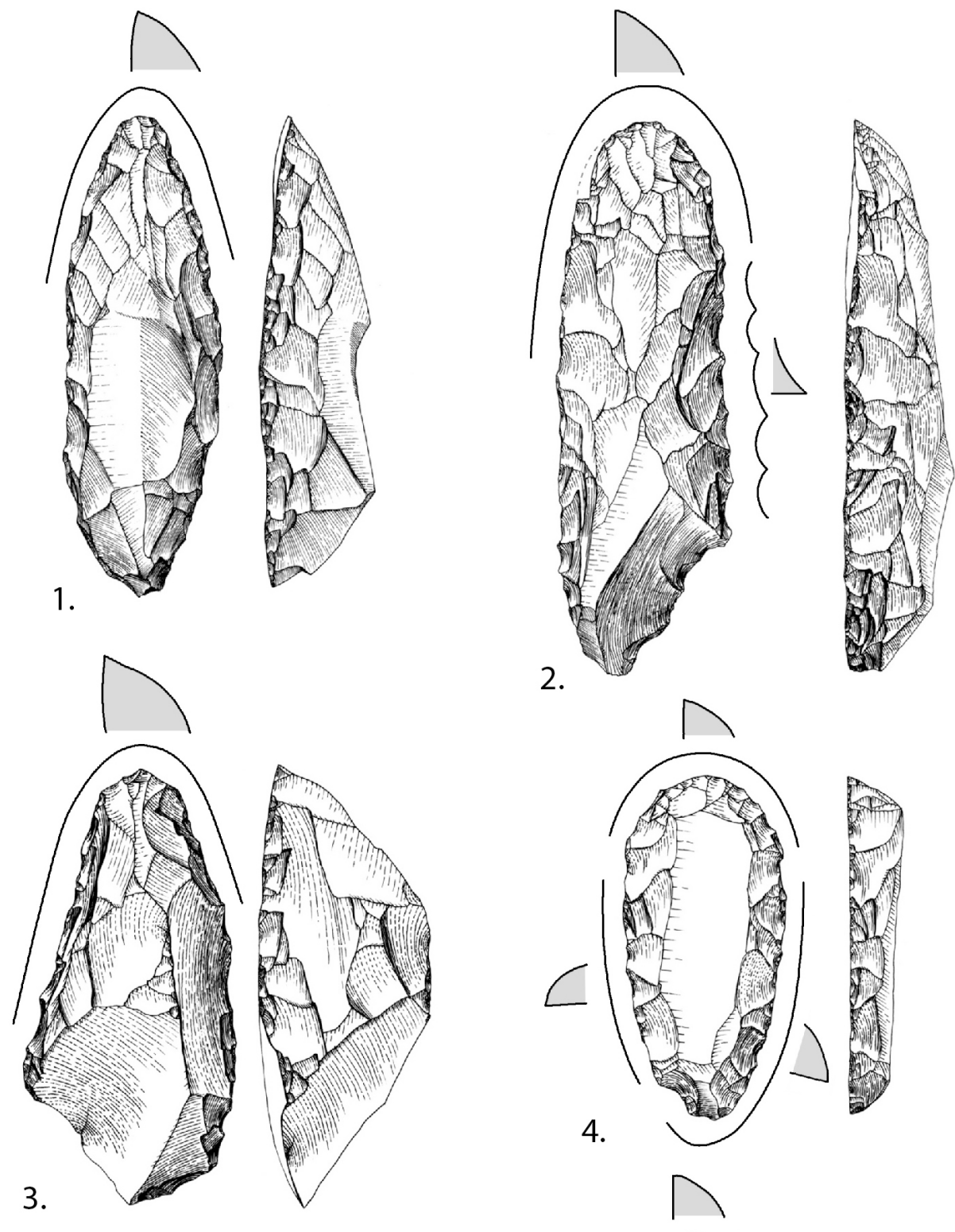

2.
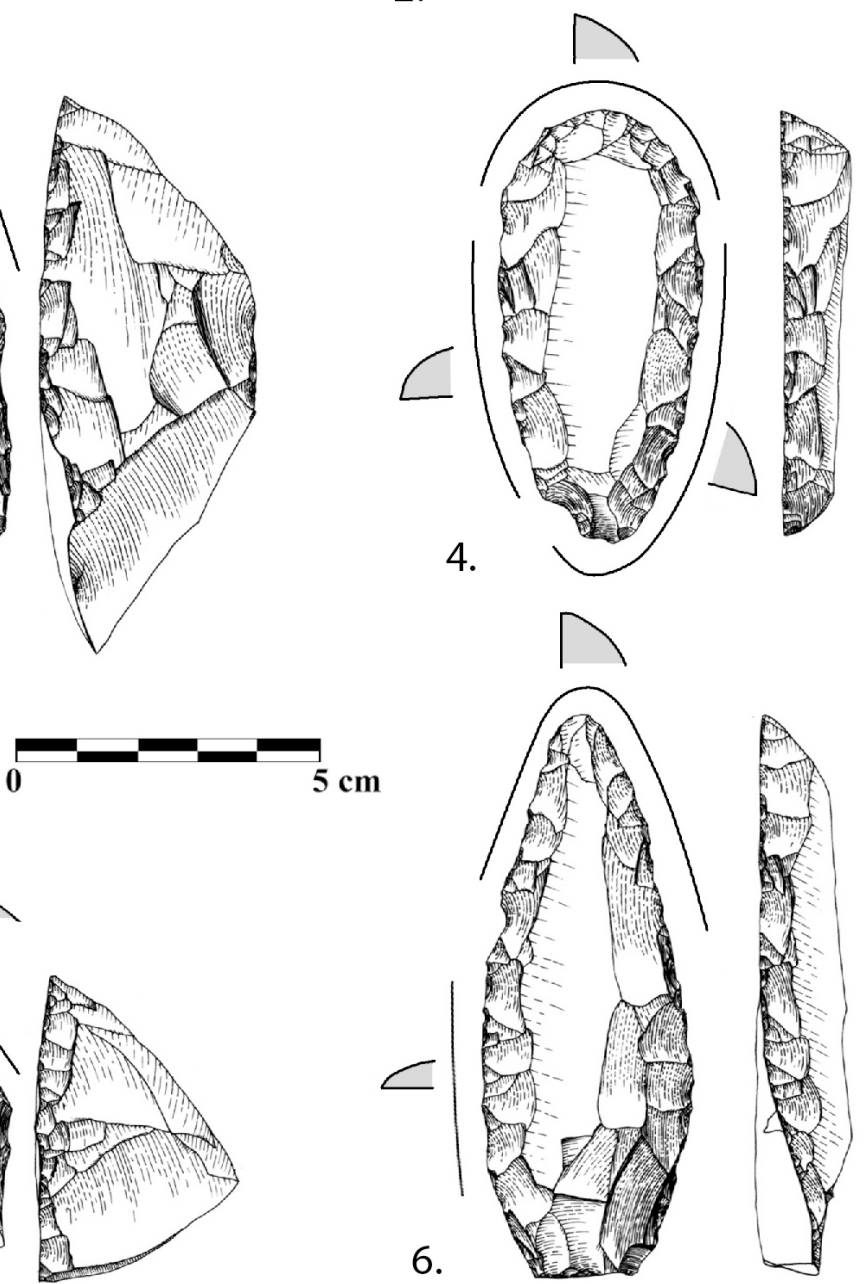

5.

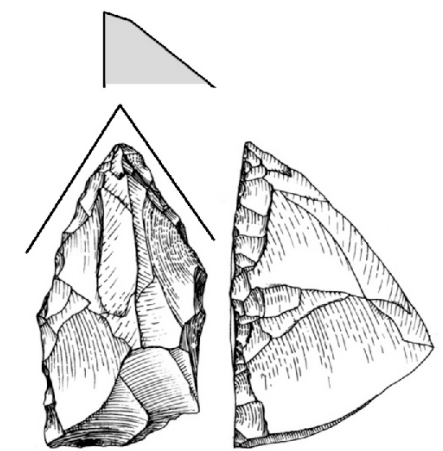

Figure 2. Exemples de pièces façonnées unifacialement du technocomplexe Itaparica provenant de GO-JA-01. La position et la délinéation de chaque partie transformative est figurée par une ligne noire, à laquelle est associée une figuration du plan de section (angles et surfaces).

Figure 2. Examples of unifacially shaped artefacts of the Itaparica technocomplex, from GO-JA-01 rockshelter. The position and delineation of each transformative part are represented by a black line, associated with a section of the edge (angles and surfaces). 


\subsection{Le corpus}

Pour aborder cet aspect des pièces façonnées unifacialement, nous nous basons sur l'importante collection mise au jour dans le site de GO-JA-01 (Etat de Goiás). Ce gisement est un abri-sous-roche fouillé entre 1975 et 1980 sous la direction de P.I. Schmitz et A.S. Barbosa (Schmitz 1987b; Schmitz et al. 1989; 2004), dont les couches inférieures sont associées au technocomplexe Itaparica. Ces couches sont datées entre 12500 et $10000 \mathrm{cal}$ BP. Elles ont livré un total de près de 40000 restes lithiques, parmi lesquels 517 outils, dont 377 pièces façonnées unifacialement, et 27 nucléus. La roche utilisée de façon prédominante est un grès silicifié fin issu d'un métamorphisme de contact entre le grès éolien de la formation Botucatu et le basalte de la formation Serra Geral qui le recouvre (formations jurassico-crétacées) (Dantas et al. 2008). Cette matière première fine est accessible en quantité aux alentours directs de l'abri sous forme de blocs et plaquettes provenant du massif gréseux dans lequel s’insère le site. Quelques petites pièces en silex de provenance indéterminée ont également été mises au jour.

\section{La gestion des pièces façonnées unifacialement dans le temps}

Un faisceau d'indices nous permet de penser que le temps d'utilisation des pièces façonnées unifacialement est long et qu'on les trouve dans différents états techniques. Le degré d'intégrité de ces artefacts en fait partie (Tableau 1). Moins d'un tiers des pièces ont été découvertes entières, alors que plus de la moitié l'ont été sous forme de fragment. Soixantesix artefacts sont en fin de vie, c'est-à-dire que les réaménagements successifs ont dénaturé la structure d'origine, si bien qu'ils ne présentent plus de potentiel fonctionnel. Enfin, des marques de reprise après cassure sont attestées sur 11 pièces.

Tableau 1. Degrés d’intégrité des pièces façonnées unifacialement de GO-JA-01.

Table 1. Integrity level of the unifacially shaped artefacts from GO-JA-01 rockshelter.

\begin{tabular}{lcccc}
\hline $\begin{array}{l}\text { Pièces entières } \\
\text { stricto sensu }\end{array}$ & $\begin{array}{c}\text { Pièces reprises après } \\
\text { cassure }\end{array}$ & $\begin{array}{c}\text { Pièces dénaturées ou en } \\
\text { fin de vie }\end{array}$ & Fragments & Total \\
\hline 110 & 11 & 66 & 190 & $\mathbf{3 7 7}$ \\
\hline
\end{tabular}

Divers procédés sont utilisés pour maintenir le potentiel fonctionnel de ces pièces après différentes phases d'utilisation. Il s'agit de raffûtages et de réaménagements, ainsi que les fractures.

\subsection{Le raffûtage}

\subsubsection{Considérations générales}

Nous entendons, par raffûtage, toute opération technique au moyen de laquelle une unité techno-fonctionnelle, altérée par une phase d'utilisation, est rendue de nouveau fonctionnelle. Le raffûtage concerne principalement (mais pas uniquement) les parties transformatives. L'étendue de cette opération peut aller d'un entretien partiel du fil jusqu'à la reprise de toute la partie transformative. Il s'agit seulement d'une reprise du bord de la pièce et en aucun cas d'une modification de sa structure. Dans le cas des pièces outil, le raffûtage ne peut avoir comme objectif que l'entretien d'une partie transformative, en mettant en place les mêmes caractères que ceux présents à l'origine, alors que dans celui d'une pièce support-d'outils, il peut permettre de conserver les caractères de la partie transformative antérieure, mais il peut tout autant les modifier sensiblement (changement d'angles, de surfaces ou de délinéations), voire mettre en place de nouveaux tranchants pour obtenir un nouvel outil (Boëda 2001). 


\subsubsection{Raffûtage des pièces façonnées unifacialement}

Les extrémités des pièces façonnées unifacialement font l'objet de différents degrés de raffûtage. Elles peuvent être reprises partiellement, par des enlèvements localisés remettant en place une partie de l'unité techno-fonctionnelle. Les états de patine de certaines pièces attestent clairement d'un tel procédé. Une autre possibilité de raffûtage, plus "radicale", a consisté à ôter entièrement l'extrémité pour ensuite la re-confectionner intégralement. Ce genre de reprise a eu lieu soit lorsque la partie transformative était dans un tel état d'exhaustion qu'elle ne pouvait plus être entretenue par des enlèvements ponctuels, soit lorsque le tailleur voulait mettre un place une partie transformative différente de la première. L'examen des déchets de production nous informe que cette dernière modalité a été mise en place selon trois procédés distincts (Figure 3).

Le raffûtage des parties latérales est également attesté par les éclats résultant de ces opérations. On différencie ces éclats de raffûtage de ceux issus de la première phase de confection par le fait qu'ils présentent un lustre d'utilisation clairement identifiable à l'œil nu au niveau de la ligne de séparation entre le talon et la face supérieure. Il s'agit d'éclats enlevés au moyen d'un coup donné sur la face plane de l'artefact. Suivant l'ampleur des modifications recherchées, cet impact est porté de façon plus ou moins éloignée du bord : la percussion peut être marginale (Figure 4) ou interne (Figure $5, \mathrm{n}^{\circ} 1$ et 2 ).

La réalisation d'enlèvements centrifuges à partir du haut de la face non plane est un autre procédé, nettement plus rare, employé pour le raffûtage des zones latérales (Figure $5, n^{\circ} 3$ et 4). L'éclat produit emporte alors toute la partie latérale de la face non plane ainsi qu'une portion de quelques millimètres d'épaisseur de la face plane.

\subsection{Les fractures et reprises après fracture}

Les fractures constituent un processus très courant de modification des pièces façonnées unifacialement au cours de leur utilisation. Ces dernières sont retrouvées dans une forte proportion sous forme fragmentaire (Schmitz et al. 2004; Fogaça 2001). Dans la collection de GO-JA-01, nous avons comptabilisé 190 fragments de ces outils contre 110 pièces entières. A la différence des raffûtages, celles-ci ne sont pas toujours provoquées de façon intentionnelle. Toutefois plusieurs indices montrent que les fractures, qu'elles soient volontaires ou accidentelles, devaient être prises en compte dans les schémas de reprise des pièces, de façon à ce que la cassure ne signifie pas nécessairement son abandon immédiat.

On trouve des fragments apicaux, mésio-apicaux, basaux, mésio-basaux et mésiaux (Figure 6 et Tableau 2). La position des fractures permet de distinguer quatre tendances : une facture unique proche de l'extrémité distale, une fracture unique proche de l'extrémité basale, une fracture unique mésiale et de multiples fractures (Figure 7). Les fragments apicaux étant de loin les plus nombreux, la fracture unique proche de l'extrémité distale est la plus courante. Mais ils sont plus de deux fois mieux représentés que les fragments mésio-basaux, leurs complémentaires. Cela implique que les fragments mésio-basaux, plus longs, devaient être réintroduits dans la sphère fonctionnelle après un affûtage de la surface de fracture en position apicale. En effet, les fractures au niveau d'une extrémité ont pour effet d'enlever cette dernière de la même façon que pour le raffûtage. 
A.
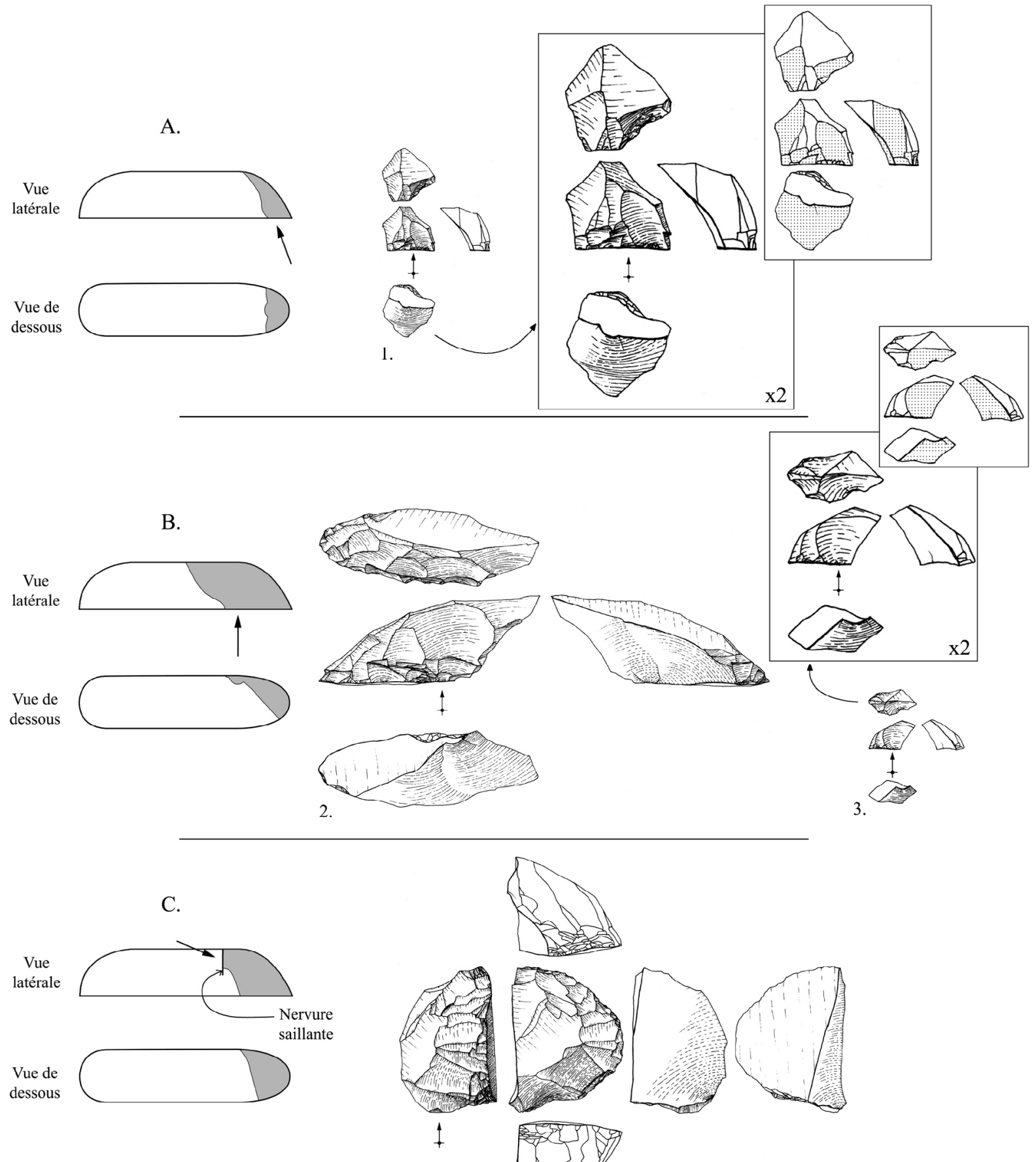

4

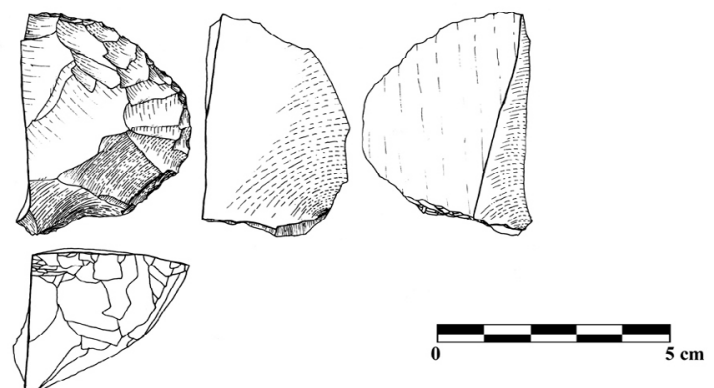

Figure 3. Procédés de raffûtage de pièces façonnées unifacialement par enlèvement complet d’une extrémité. A : coup porté à l'extrémité, sur la face plane et dans l'axe longitudinal ; B : coup porté sur la face plane, au niveau d'un bord, à proximité de l'extrémité ; C : coup tangentiel à la surface de la pièce porté sur la face non plane sur une nervure saillante à proximité de l'extrémité. Les pièces 1 et 3 présentent une double patine (patine récente en surfaces pointillées sur les schémas) attestant de raffûtages antérieurs plus légers (1) et d’un premier essai avorté de retrait de l'extrémité (3).

Figure 3. Resharpening processes of unifacially shaped artefacts by removing an end. A: blow to the end, on the plane surface and in the longitudinal axis; B: blow to the plane surface in one side, near the end; C: tangential blow to a prominent ridge on the non-plane surface, near the end. Artefacts 1 and 3 have a double patina (recent patinas are represented by dotted surfaces in the drawings) that demonstrate resharpening (1) and a first aborted tentative of removal of the end (3). 


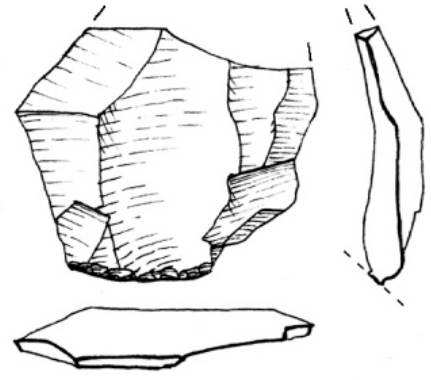

1.

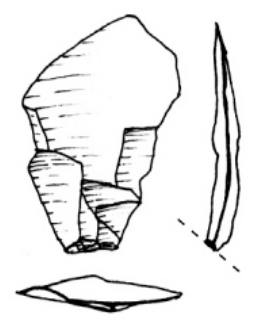

2.

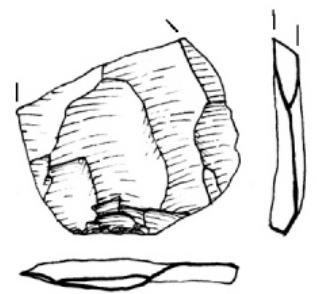

3.

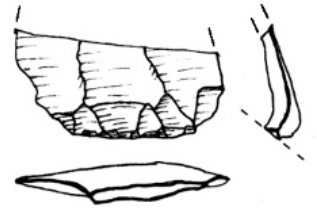

4.

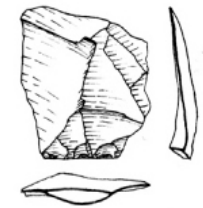

5.

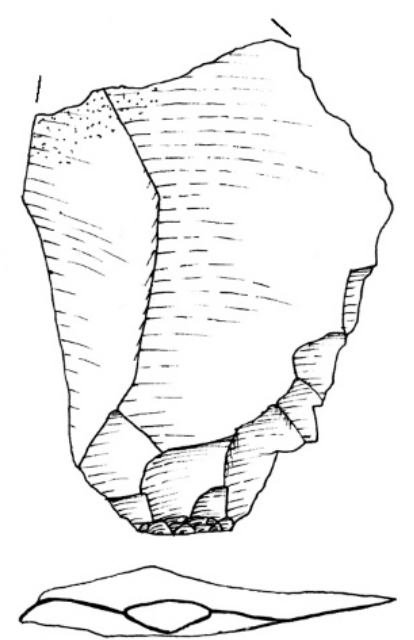

6.

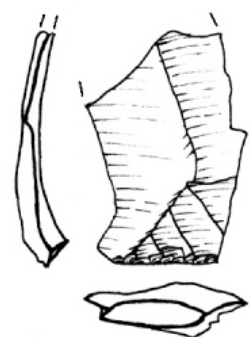

9.
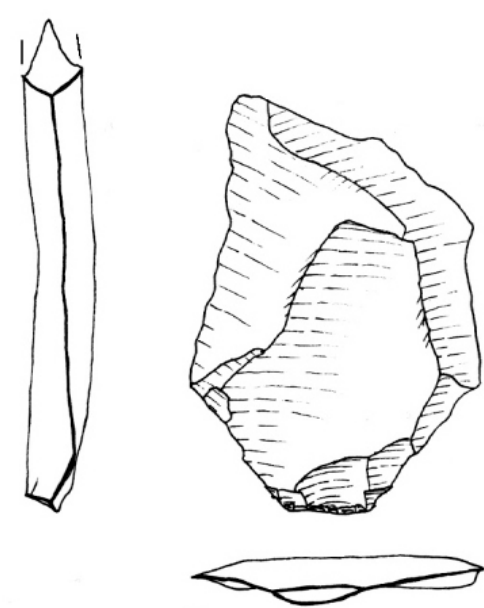

7.

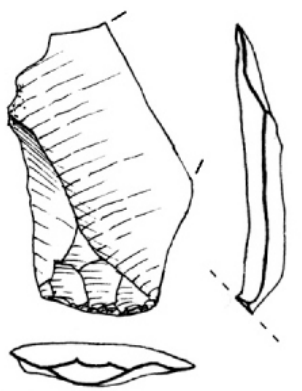

10.

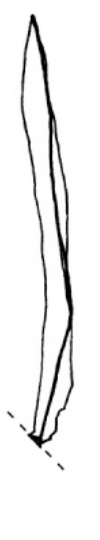

8 .
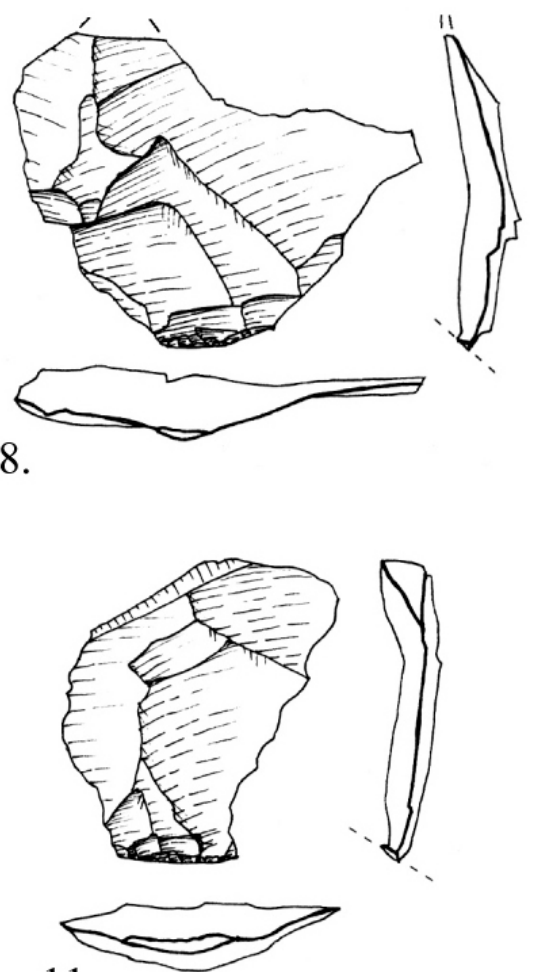

11.

Figure 4. Eclats de raffûtage latéral de pièces façonnées unifacialement détachés par percussion marginale. Tous ces éclats présentent un lustre d’utilisation identifiable à l'œil nu au niveau de la ligne de séparation entre le talon et la face supérieure.

Figure 4. Lateral resharpening flakes from unifacially shaped artefacts removed by marginal percussion. Every flake presents a use luster visible to the naked eye in the line between the butt and the external face. 


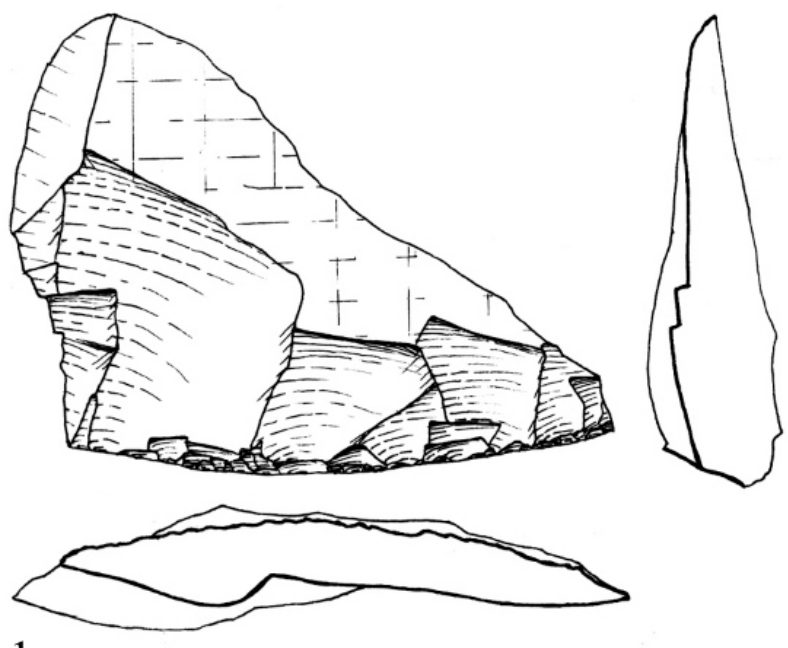

1.
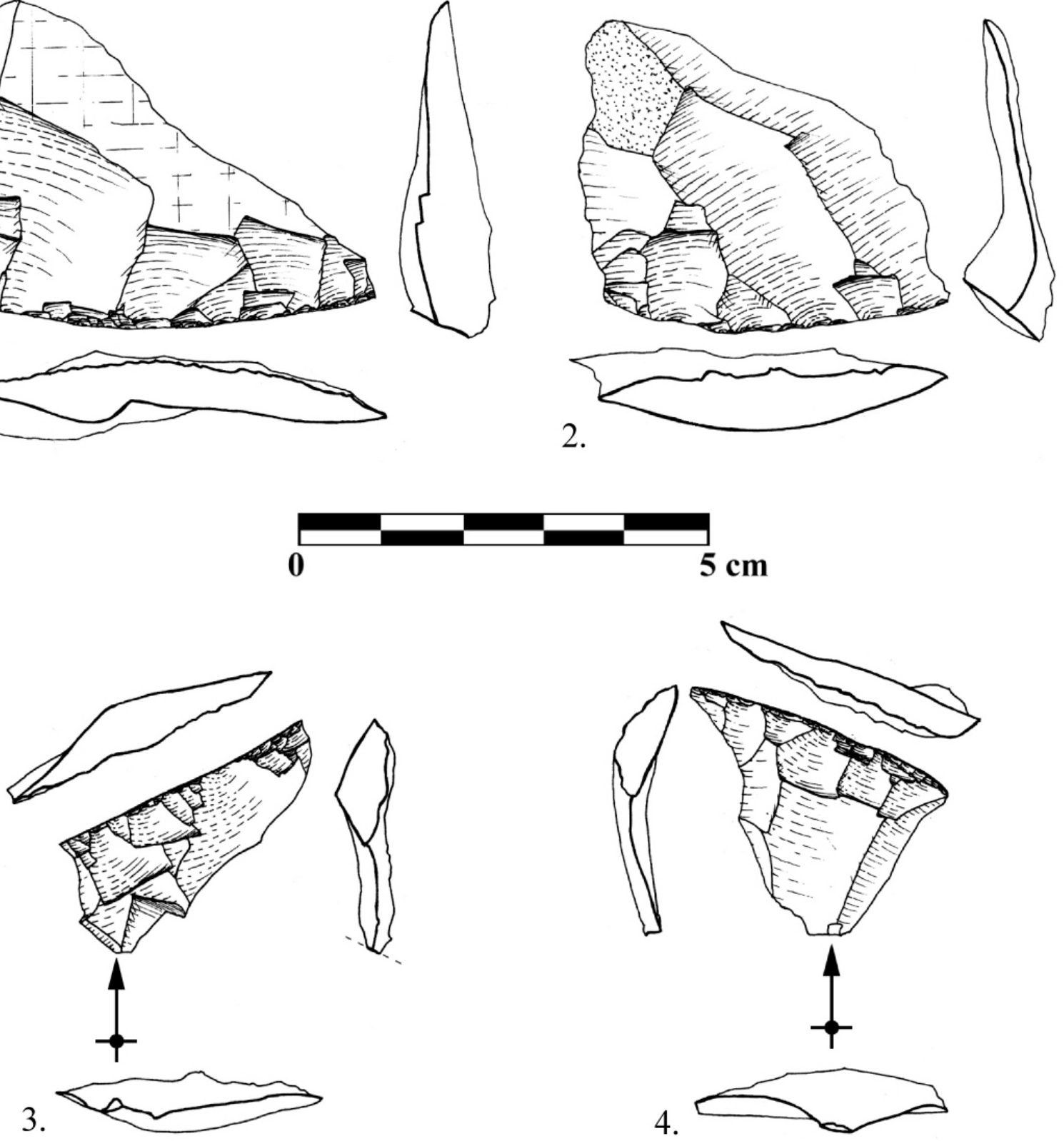

Figure 5. Eclats de raffûtage latéral de pièces façonnées unifacialement. Les pièces 1 et 2 sont détachées par percussion interne depuis la face plane et présentent un lustre d'utilisation identifiable à l'œil nu au niveau de la ligne de séparation entre le talon et la face supérieure. Les pièces 3 et 4 sont détachées par percussion marginale de façon centrifuge, depuis le haut de la face non plane.

Figure 5. Lateral resharpening flakes from unifacially shaped artifacts. Artefacts 1 and 2 were removed par internal percussion from the plane surface and present a use luster visible to the naked eye in the line between the butt and the external face. Artefacts 3 and 4 were removed by marginal percussion in a centrifugal direction, from the top of the non-plane surface. 


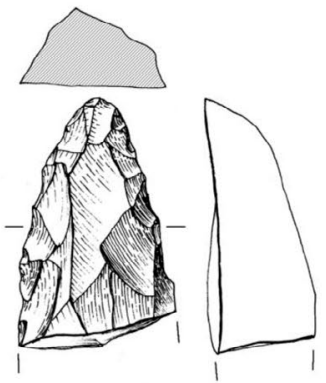

a.

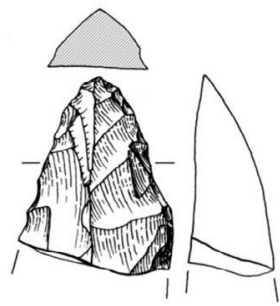

d.

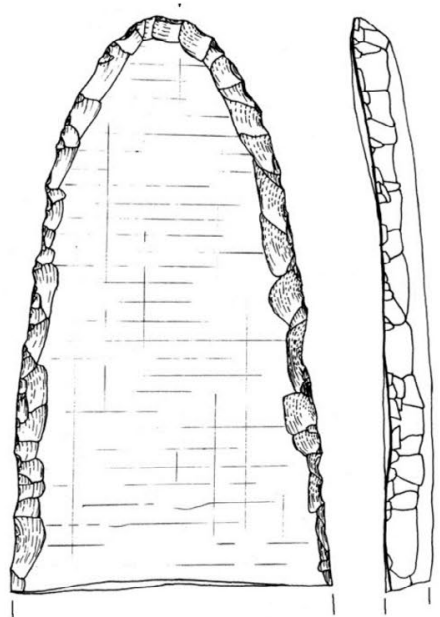

h.

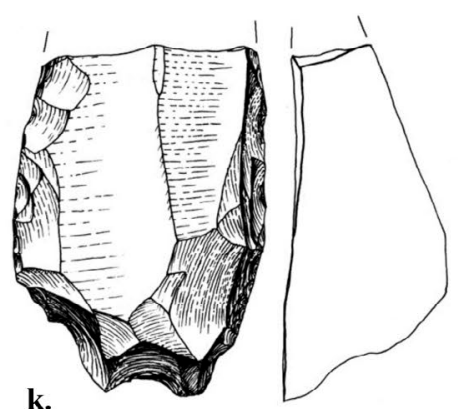

k.
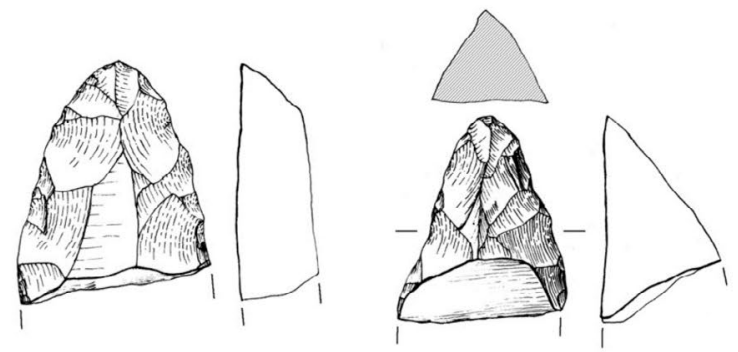

f.
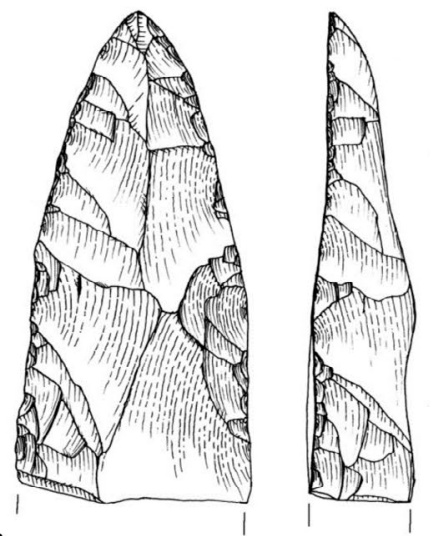

i.

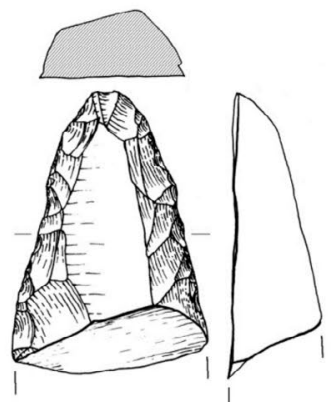

b.

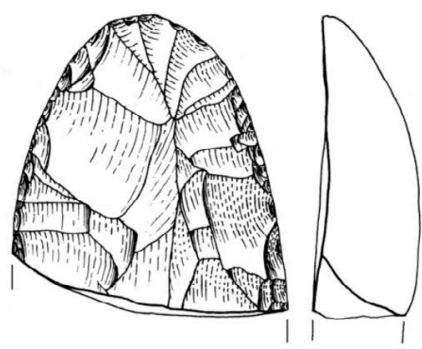

g.
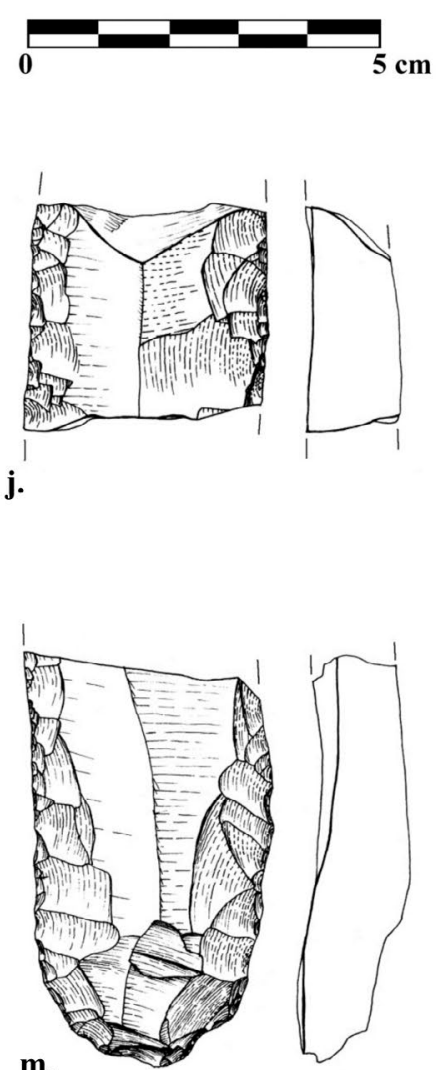

m.

Figure 6. Fragments de pièces façonnées unifacialement. a-g : fragments apicaux, h-i : fragments mésio-apicaux, $\mathrm{j}$ : fragment mésial, k-m : fragments mésio-basaux.

Figure 6. Unifacially shaped artefact fragments. a-g: apical fragments, h-i: mesio-apical fragments, j: mesial fragment, k-m: mesio-basal fragments. 
Tableau 2. Décompte des fragments de pièces façonnées unifacialement à GO-JA-01.

Table 2. Count of the unifacially shaped artifacts fragments from GO-JA-01 rockshelter.

\begin{tabular}{ll}
\hline Fragments apicaux & $\mathbf{7 3}$ \\
\hline Fragments basaux & 16 \\
Fragments mésio-apicaux & 29 \\
Fragments mésio-basaux & 30 \\
Fragments mésiaux & 26 \\
Extrémités indéterminées & 16 \\
\hline TOTAL & $\mathbf{1 9 0}$ \\
\hline
\end{tabular}

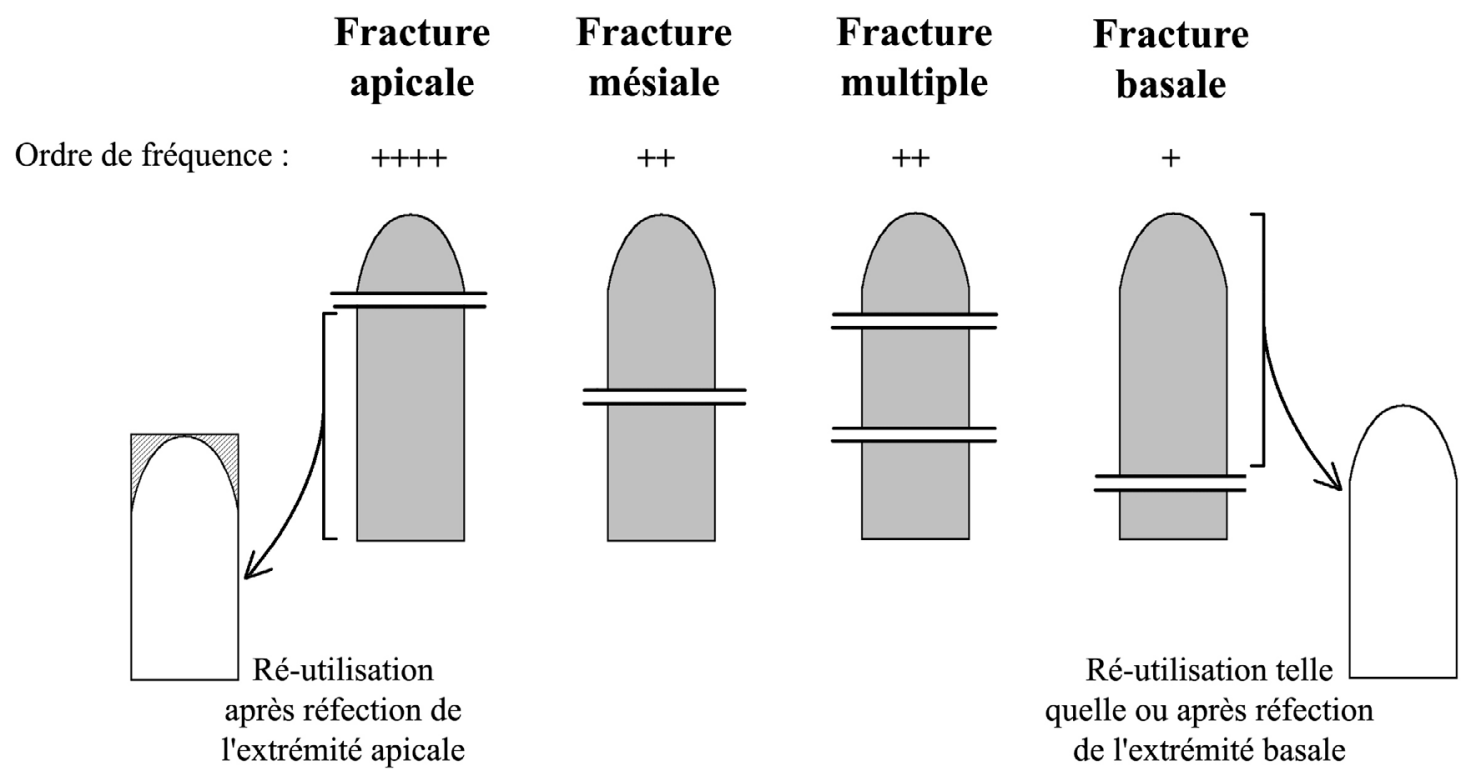

Figure 7. Les principaux schémas de fracturation des pièces façonnées unifacialement à GO-JA-01 et leur ordre de fréquence.

Figure 7. Frequencies of the main fracturing schemes of unifacially shaped artefacts from GO-JA-01 rockshelter.

Par ailleurs, lorsque c'est l'extrémité basale qui est fracturée, il est envisageable que les fragments mésio-apicaux résultant de ces fractures (par exemple Figure $6, \mathrm{~h}$ et i) aient été utilisés tels quels. Ils présentaient effectivement encore les caractères fonctionnels essentiels des pièces façonnées unifacialement et en particulier la partie transformative apicale. Un cas de légère reprise après une fracture basale est également attesté à GO-JA-01 (Figure 8.e). Etant donné le nombre réduit de fragments basaux de pièces façonnées unifacialement retrouvés dans les collections, ce type de fracturation ne devait avoir lieu qu'assez rarement.

Quand les fractures se développent vers le milieu de la pièce, la modification subie par l'outil est alors plus lourde de conséquences volumétriques. Les fragments repris ont perdu une grande partie de leur allongement initial. D’après les décomptes des fragments (Tableau 2), les fractures dans la zone mésiale sont moins fréquentes que celles situées à l'extrémité apicale. Il est possible que cette contrainte imposée par la fracturation mésiale ait souvent conduit les utilisateurs de ces pièces à les abandonner après de telles cassures. Cette supposition est soutenue par le fait que l'on trouve, dans la collection de GO-JA-01, autant de fragments mésio-basaux que de fragments mésio-apicaux. Toutefois, comme l'attestent certaines pièces, au moins une partie de ces fragments a été reprise en vue d'une nouvelle utilisation, et ce malgré la perte d'allongement de l'artefact (Figure 8. a à d). Ces reprises consistent uniquement en un réaménagement localisé d'un côté ou des deux côtés de la surface de fracture. 

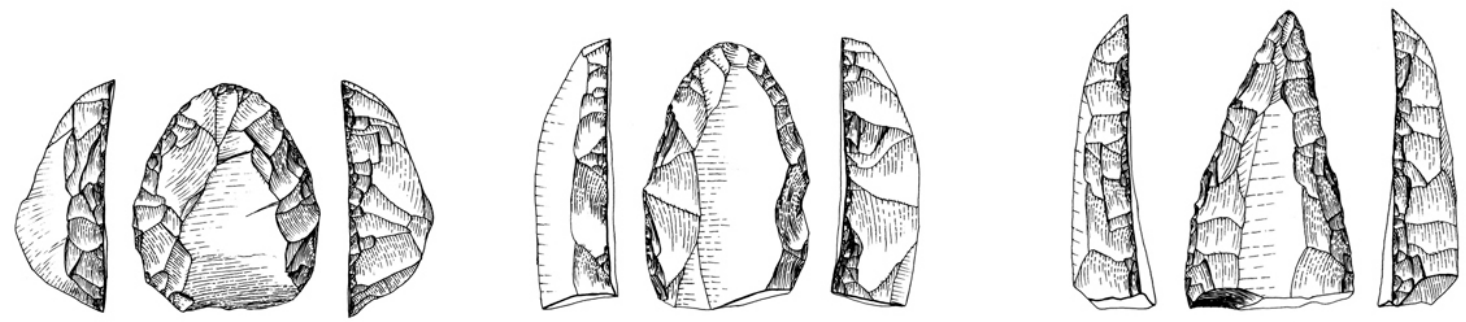

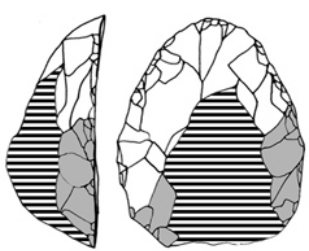

a.
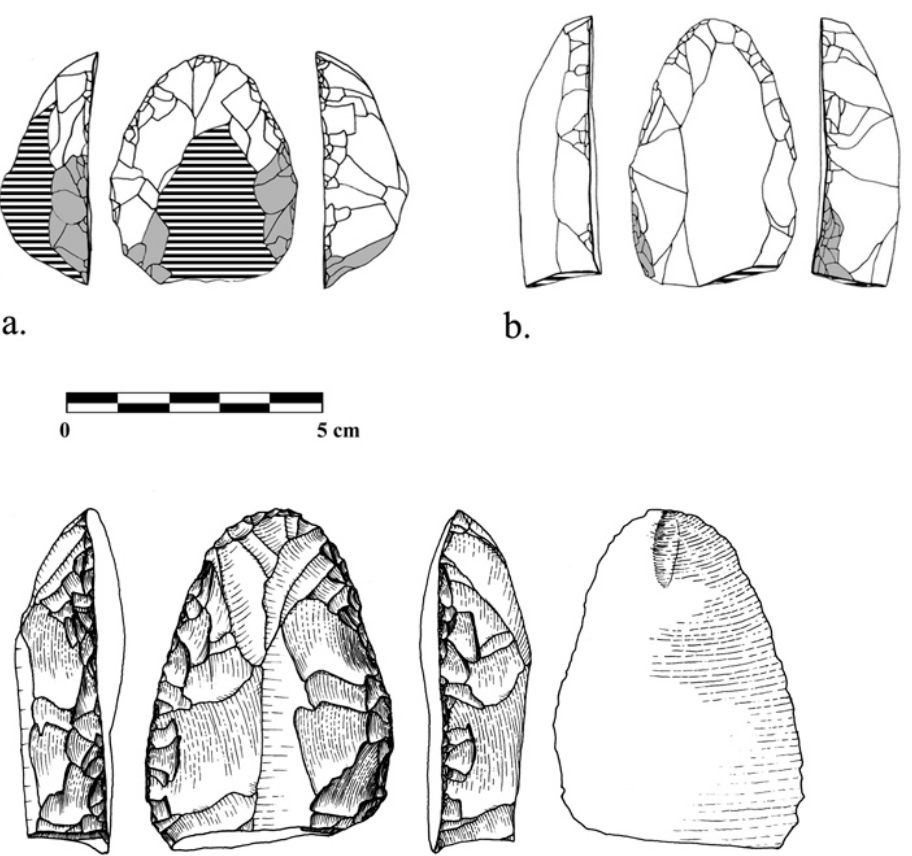

b.
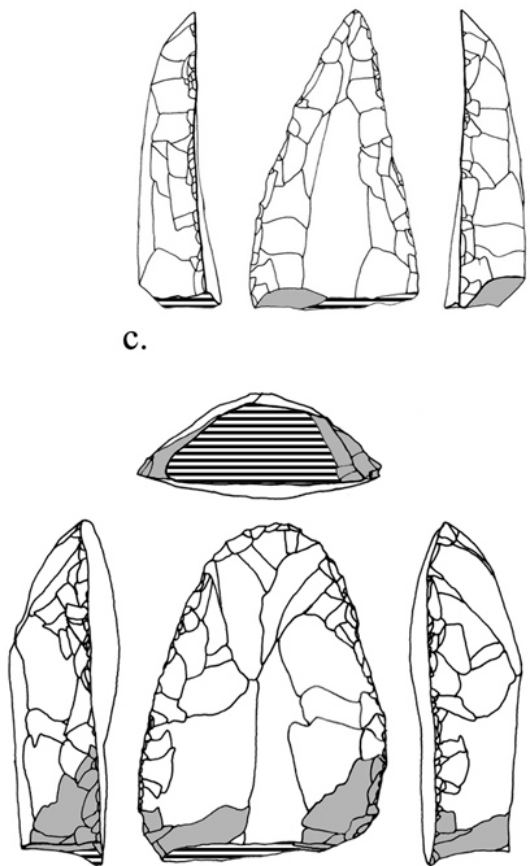

d.

e.
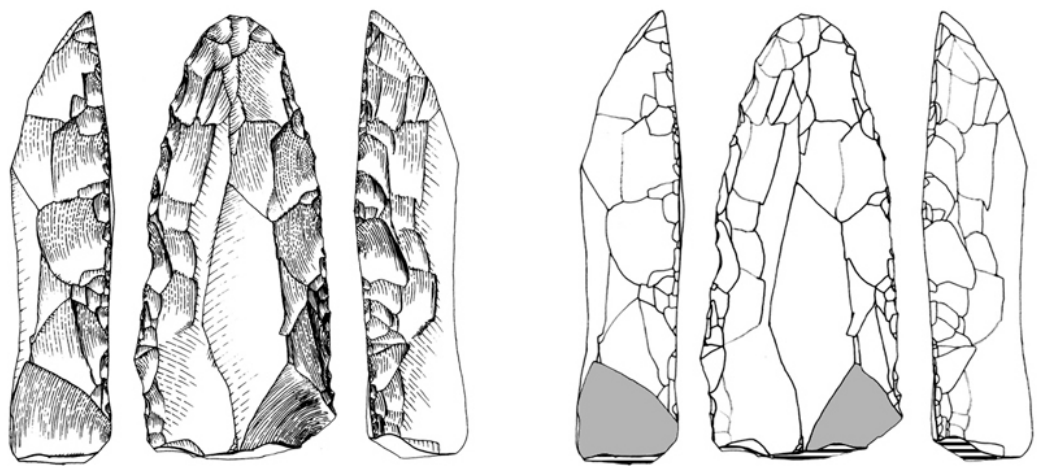

Figure 8. Pièces façonnées unifacialement reprises après fracture. Légende des schémas : $1:$ surface de fracture, 2 : négatif d'enlèvement postérieur à la fracture.

Figure 8. Unifacially shaped artefacts resumed after breaking. Captions: 1: breaking surface, 2: removal after breaking.

Le volume de ces pièces reprises après fracture mésiale est comparable à celui de pièces façonnées unifacialement issues d’une ou plusieurs phases de réaménagement.

\subsection{Le réaménagement}

\subsubsection{Définitions}

Le réaménagement d'une pièce façonnée unifacialement constitue une modification sensible de l'artefact d'origine après une ou plusieurs phases d'utilisation. Cette reprise ne se 
limite pas à rendre les parties actives à nouveau fonctionnelles. Il s'agit d'un re-façonnage, partiel ou complet, de la pièce qui mène à une transformation plus ou moins importante de la structure volumétrique initiale.

A force de réaménagements, la réserve de matière constituée par la pièce peut arriver à exhaustion. La fin de vie des pièces façonnées unifacialement conduit parfois à la dénaturation de ces dernières. Par dénaturation, nous entendons une étape de reprise majeure, qui aboutit à la perte d'un ou plusieurs des critères essentiels de la structure volumétrique ou fonctionnelle de la pièce (Boëda 1997; Soriano 2000). Dans le cas des pièces façonnées unifacialement, il peut s'agir, par exemple, d'une perte d'allongement ou d'une disparition de la partie transformative d'extrémité apicale. L'étape ultime de la dénaturation d'un artefact est l'altération de tout son potentiel fonctionnel. La pièce n'a alors plus aucune partie transformative dans un état opérationnel.

\subsubsection{Indices de réaménagements}

L’observation détaillée des schémas diacritiques nous fournit quelques indices quant à l'existence d'un état antérieur nous laissant supposer la mise en œuvre d'un réaménagement. Ces indices sont notamment la réalisation d'un ou plusieurs grands enlèvements qui rompent la symétrie d'origine de la pièce ou l'exécution d'une nouvelle phase de façonnage de tout le côté d'une pièce. En prenant en compte les différences de patine sur la surface des artefacts, on peut reconnaître des cas de re-façonnage partiel d'une partie de la face non plane (Figure 9 . a). Il arrive que les enlèvements de réaménagement soient effectués depuis le haut de la face non plane, selon une direction centrifuge (Figure 9. b). Ce type de reprise a lieu sur des pièces épaisses, dont les angles des côtés sont trop ouverts pour permettre un nouveau façonnage par des enlèvements centripètes. Les différences de patine permettent aussi de repérer un refaçonnage presque complet de la face non plane (Figure 9. c, d, e).

\subsubsection{Pièces dénaturées}

Les pièces façonnées unifacialement dénaturées sont présentes dans des proportions non négligeables dans les collections archéologiques. A GO-JA-01, on en dénombre 66. Sur le plan volumétrique, deux grandes catégories se distinguent : les pièces peu ou pas allongées (Figure 10, n 1 et 3-7), au nombre de 49 sur 66 et pour lesquelles l'allongement original a été perdu suite à une ou plusieurs phases de fracture ou de réaménagement (parfois, ces modifications sont si profondes qu'il est difficile de retrouver l'axe d'allongement original de l'artefact (par exemple, Figure $10, n^{\circ} 4$ et 7$)$ ) ; les pièces allongées, plus rares (17 sur 66 pièces dénaturées) et dont la structure est assez proche de celle des pièces non dénaturées, mais leurs bords ou leurs extrémités ne possèdent plus de potentiel fonctionnel opérationnel (Figure 10, $\mathrm{n}^{\circ} 2$ ).

C’est à partir des pièces dénaturées que nous pouvons tenter une définition des schémas de réaménagement. Ces dernières donnent une idée de l'état ultime des pièces, une fois que le processus de réaménagement est parvenu à exhaustion. L'existence de ces deux catégories volumétriques distinctes se traduit par une organisation bimodale de l'histogramme des rapports longueur/largeur des pièces dénaturées de GO-JA-01 (Figure 11). Il est intéressant de remarquer que les classes de rapport longueur/largeur les moins représentées pour les pièces dénaturées (de 2,2 à 2,6) correspondent précisément à celles où les pièces entières non dénaturées sont les plus nombreuses. On peut en conclure que les opérations de réaménagement se traduisent soit par une réduction de l'allongement original des pièces soit, mais dans une bien moindre mesure, par une augmentation de cet allongement original. 

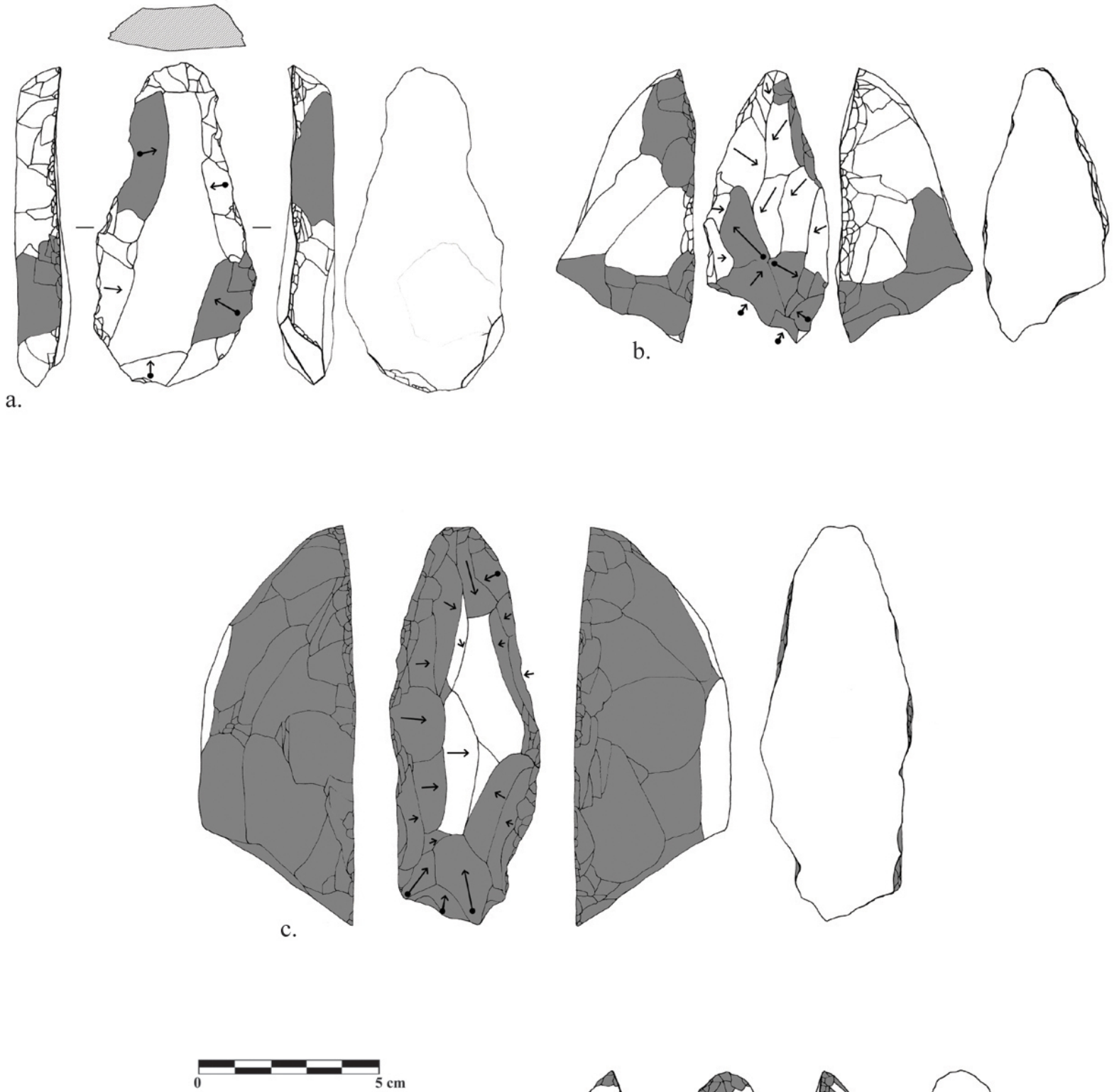

d.
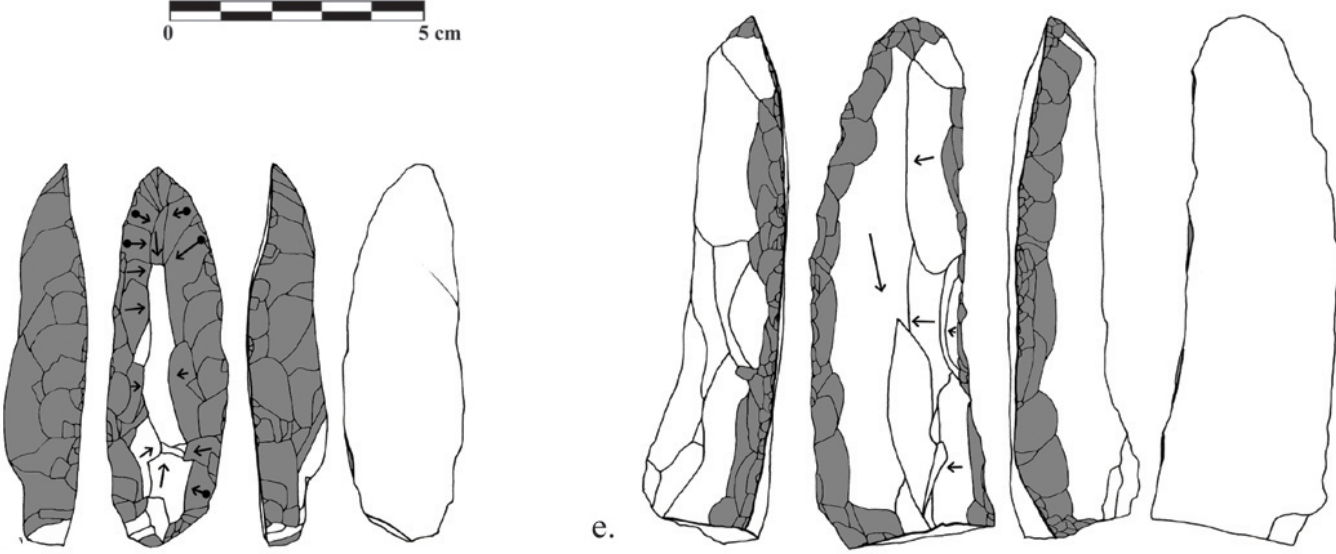

Figure 9. Exemples de pièces présentant des doubles patines attestant d’opérations de réaménagement. En gris : les négatifs présentant une patine récente.

Figure 9. Examples of artefacts with double patinas arising from reconfigurations. In grey: removals with a recent patina. 

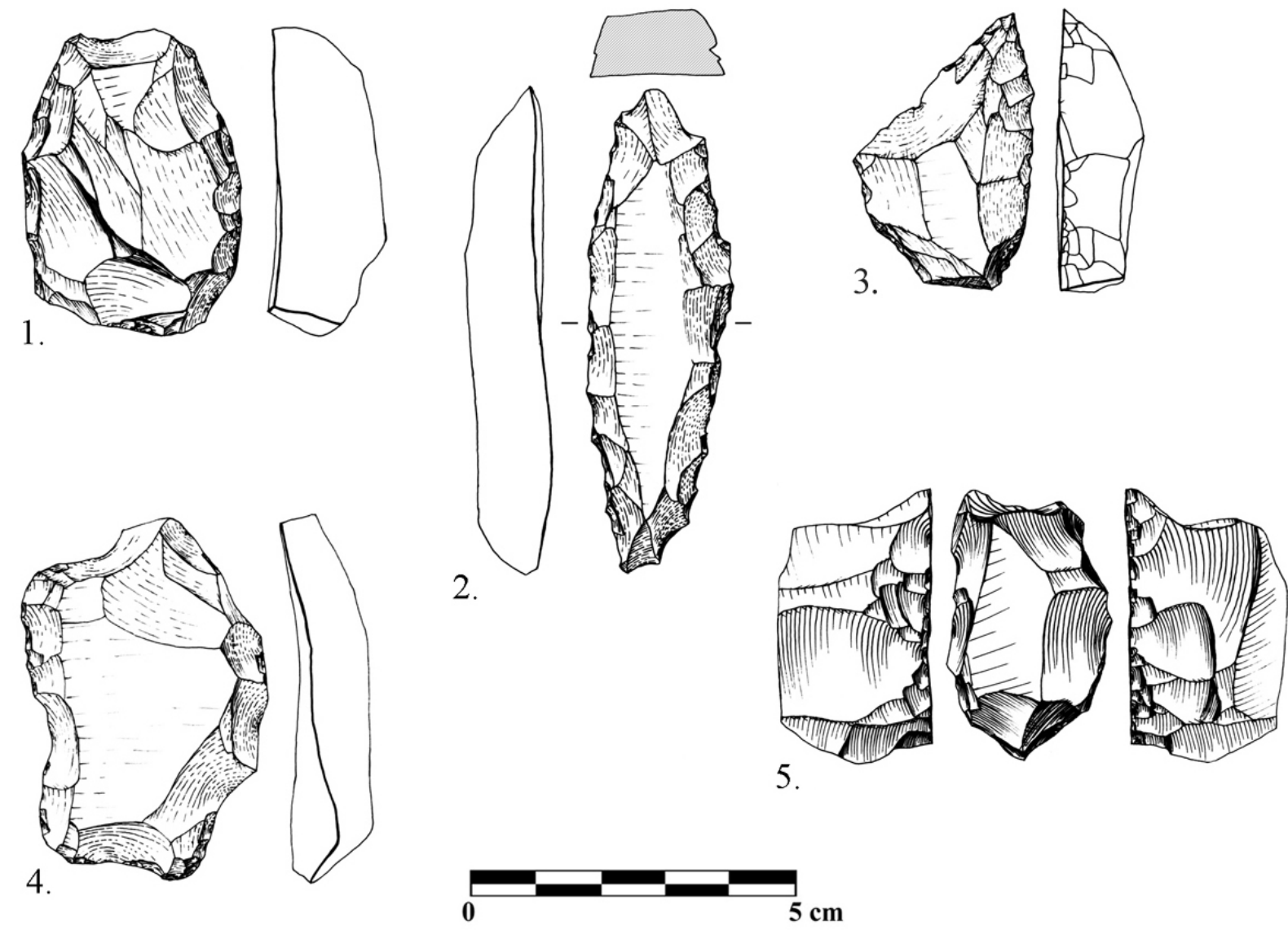

4.
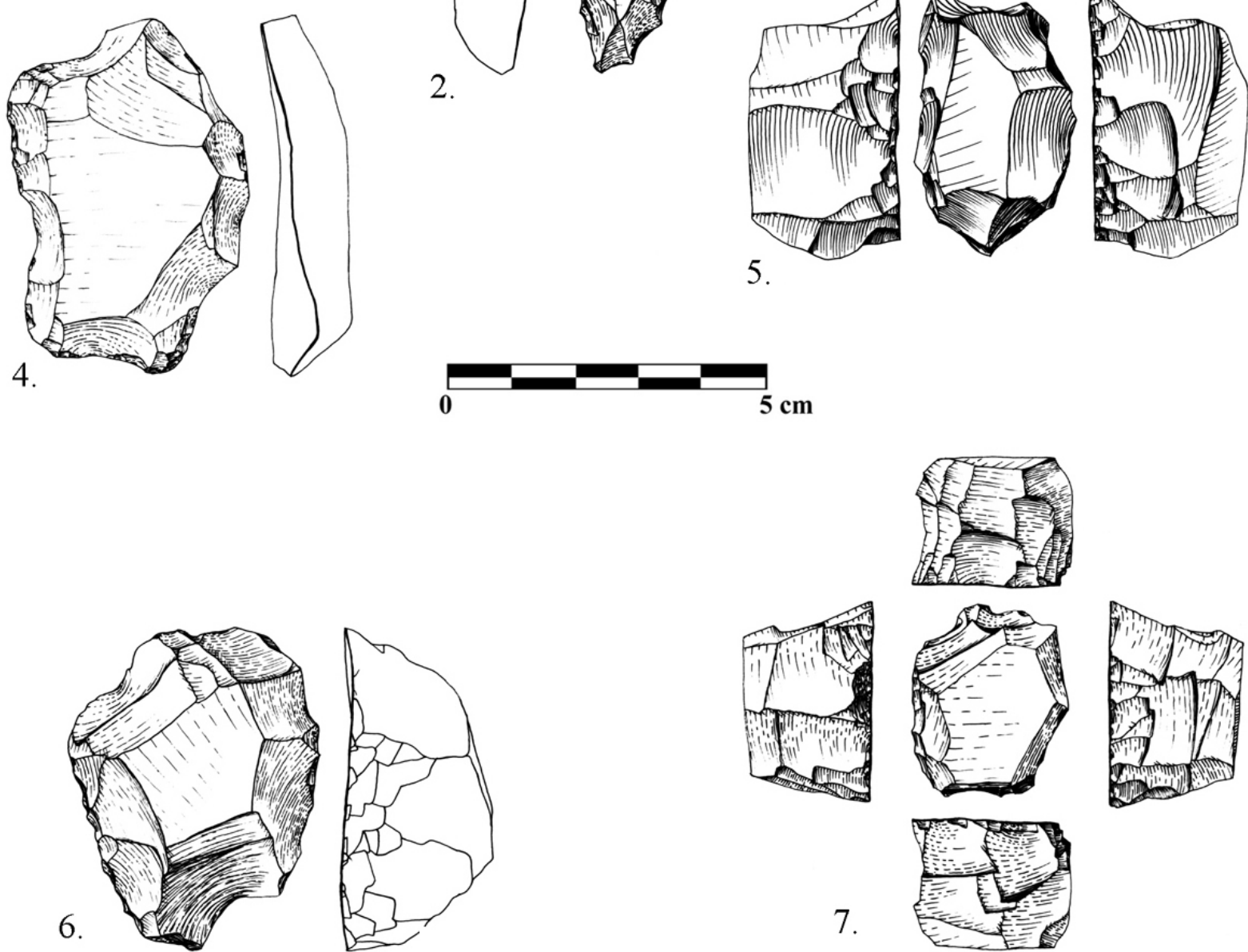

Figure 10. Exemples de pièces façonnées unifacialement dénaturées.

Figure 10. Examples of exhausted unifacially shaped artefacts.

En revanche, les épaisseurs des pièces dénaturées présentent la même variabilité que celles des pièces entières (Figure 12), les opérations de réaménagement n’ayant donc pas ou peu d'incidence sur les épaisseurs. 


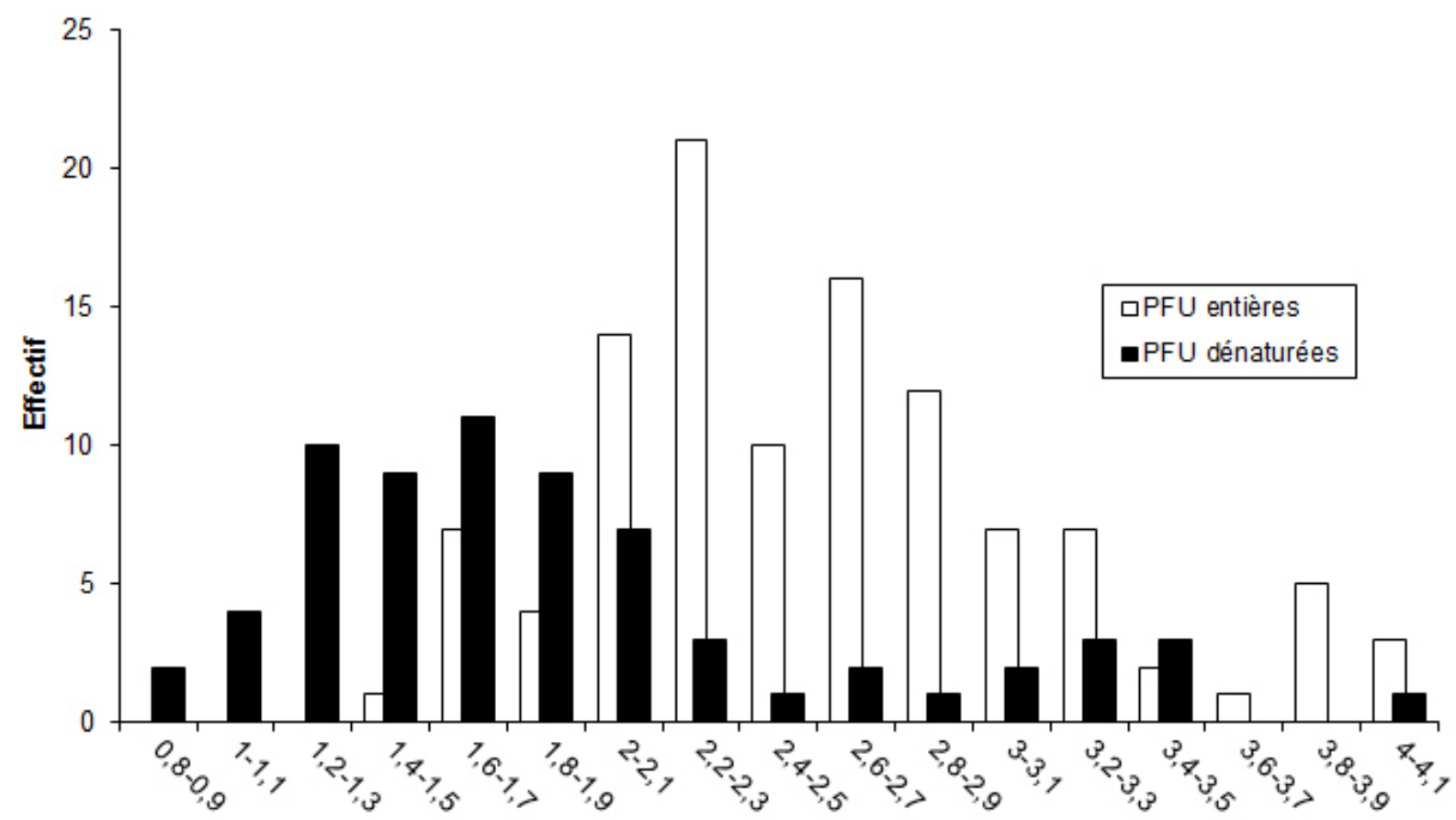

Rapport longueur / largeur

Figure 11. Histogramme du rapport longueur/largeur des pièces façonnées unifacialement de GO-JA-01. Figure 11. Histogram of unifacially shaped artefacts from GO-JA-01 rockshelter length/width ratio.

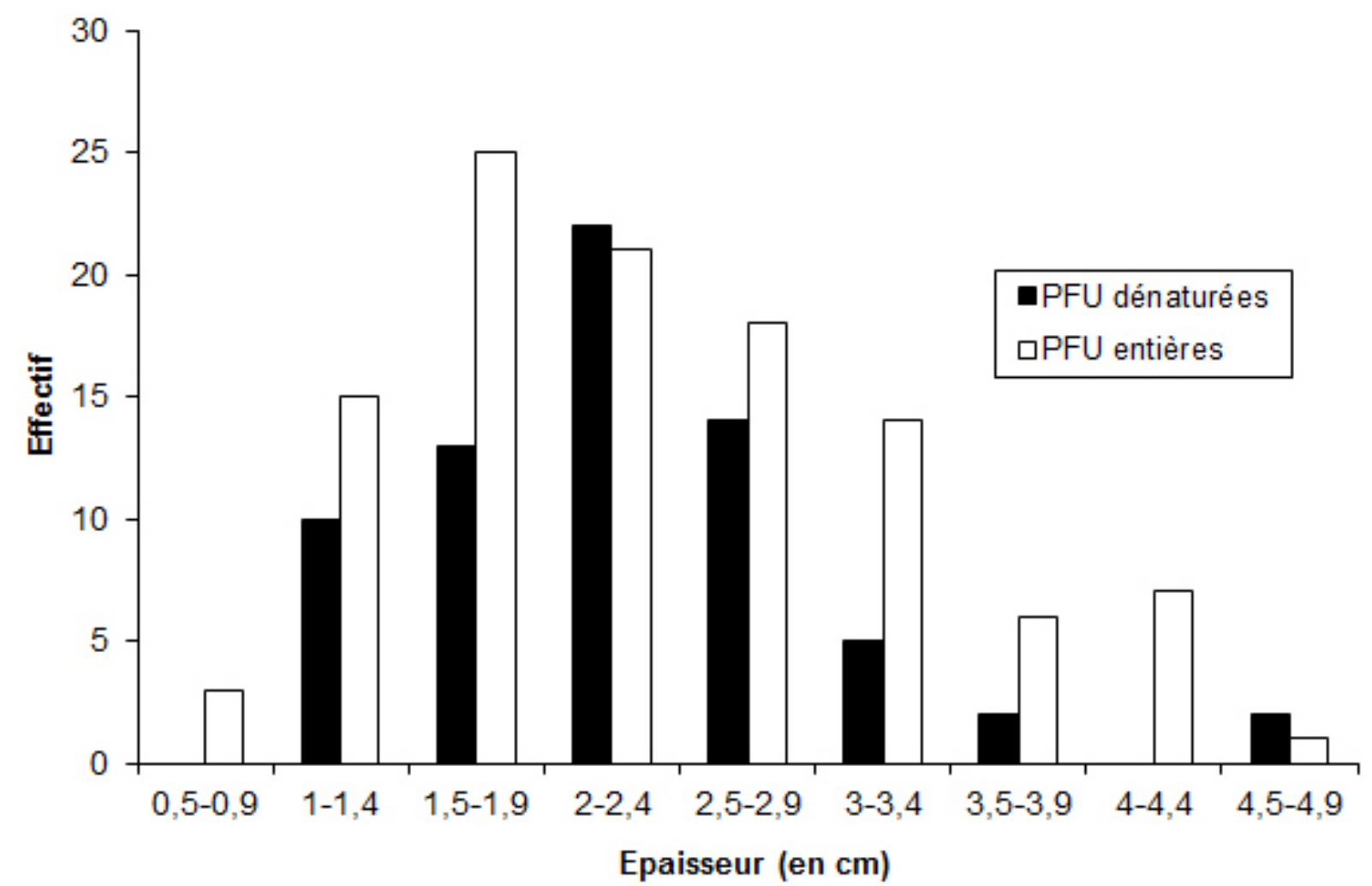

Figure 12. Histogramme des épaisseurs des pièces façonnées unifacialement de GO-JA-01.

Figure 12. Histogram of thickness of unifacially shaped artefacts from GO-JA-01 rockshelter. 


\subsubsection{Synthèse}

Les réaménagements paraissent donc constants au cours de la vie des pièces façonnées unifacialement, et ce jusqu'à leur dénaturation. L’observation des indices de réaménagement et des pièces dénaturées nous amène à distinguer deux schémas principaux :

- SCHEMA I : il consiste en une reprise portant essentiellement sur les extrémités. Il conduit à une diminution de la longueur des pièces, c'est-à-dire à une diminution du rapport longueur/largeur. C'est le schéma dominant parmi les pièces de GO-JA-01.

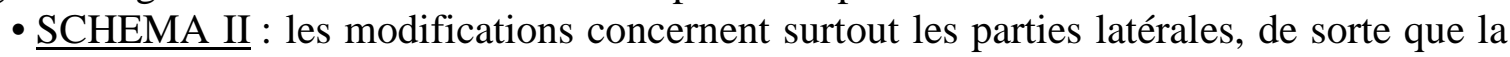
pièce garde une longueur identique ou presque à celle de son état antérieur. Ce schéma conduit à une diminution de la largeur des pièces, autrement dit à une augmentation du rapport longueur/largeur. Il s'agit d'un schéma nettement minoritaire par rapport au précédent.

Ces deux schémas correspondent de près à ceux décrits par E. Fogaça $(2001 ; 2003)$ à partir des pièces de la Lapa do Boquete.

\section{Conclusion et discussion}

\subsection{La gestion des pièces façonnées unifacialement dans le temps}

Les pièces façonnées unifacialement du technocomplexe Itaparica s’inscrivent dans un processus technique long, durant lequel leur potentiel fonctionnel évolue, de même que leurs propriétés structurelles. Suite à sa production, l'artefact subit sa première phase d'utilisation, au cours de laquelle un ou plusieurs raffûtages peuvent avoir lieu, tant à l'extrémité de la pièce qu'au niveau de ses parties latérales. La succession de plusieurs cycles d'utilisation et raffûtage peut amener cet outil jusqu'à fracturation ou épuisement de son potentiel fonctionnel (Figures 13 et 14). La pièce est alors soit abandonnée, soit réaménagée de telle sorte que l'allongement de la structure est maintenu (schéma II), soit réaménagée par une réduction des extrémités et donc une diminution du rapport longueur/largeur (schéma I), cette dernière solution étant de loin la plus employée. Une fois ce ou ces réaménagements opérés, la pièce est de nouveau utilisée et raffûtée une ou plusieurs fois. Cela amène à une fracturation ou à l'épuisement du potentiel fonctionnel de la pièce. L'artefact est alors abandonné ou repris selon les mêmes modalités que celles précédemment exposées. Plusieurs phases de réaménagement peuvent se succéder de la sorte, l'étape ultime de la vie utile de ces artefacts étant leur dénaturation définitive suivie par leur abandon.

Les pièces façonnées unifacialement sont donc des artefacts conçus pour être opérationnels pendant une longue durée. Leur structure est réalisée de façon à supporter un fort potentiel de raffûtage et de réaménagement.

\section{2. À propos des facteurs de variabilité des pièces façonnées unifacialement}

Dans son étude du matériel de la Lapa do Boquete, E. Fogaça conclut que les processus de réaménagement des pièces façonnées unifacialement sont à l'origine de leur variabilité. Selon son interprétation, les pièces mises au jour dans ce site auraient été abandonnées à différents moments de leur vie et c'est leur degré d'exhaustion plus ou moins important qui expliquerait leurs différences (Fogaça 2001: 281, 414). L’approche quantitative de cette catégorie d'artefacts, permise par la richesse exceptionnelle de la collection de pièces façonnées unifacialement mises au jour à GO-JA-01, nous amène à modérer ces conclusions.

Comme nous venons de le montrer, il est indéniable qu’il existe différents états de vie de ces supports. Les proportions considérables de fragments et de pièces dénaturées en sont les témoins. Toutefois, il ne nous paraît pas envisageable d'expliquer la totalité de la variabilité des pièces façonnées unifacialement de GO-JA-01 par le fait qu'il s'agit de pièces dans 
différents états d'exhaustion. Cette variation “diachronique” ne touche pas ou peu la structure originale des pièces (Lourdeau 2010). Les réaménagements ont des implications au niveau des dimensions ou de la morphologie des artefacts, mais leurs conséquences au niveau du volume et probablement de leurs fonctionnements sont plus modérées. Ainsi, un critère tel que la section transversale ne paraît pas avoir été modifié de façon notoire au cours des différentes phases de réaménagement. Les proportions des trois catégories de section sont équivalentes pour les pièces entières et pour les pièces dénaturées (Tableau 3). Les pièces dénaturées à section trapézoïdale ont gardé leur grand négatif central plan, antérieur au débitage de l'éclat-support. Ainsi, les pièces à une section trapézoïdale ont conservé ce caractère volumétrique de leur premier façonnage à leur abandon.

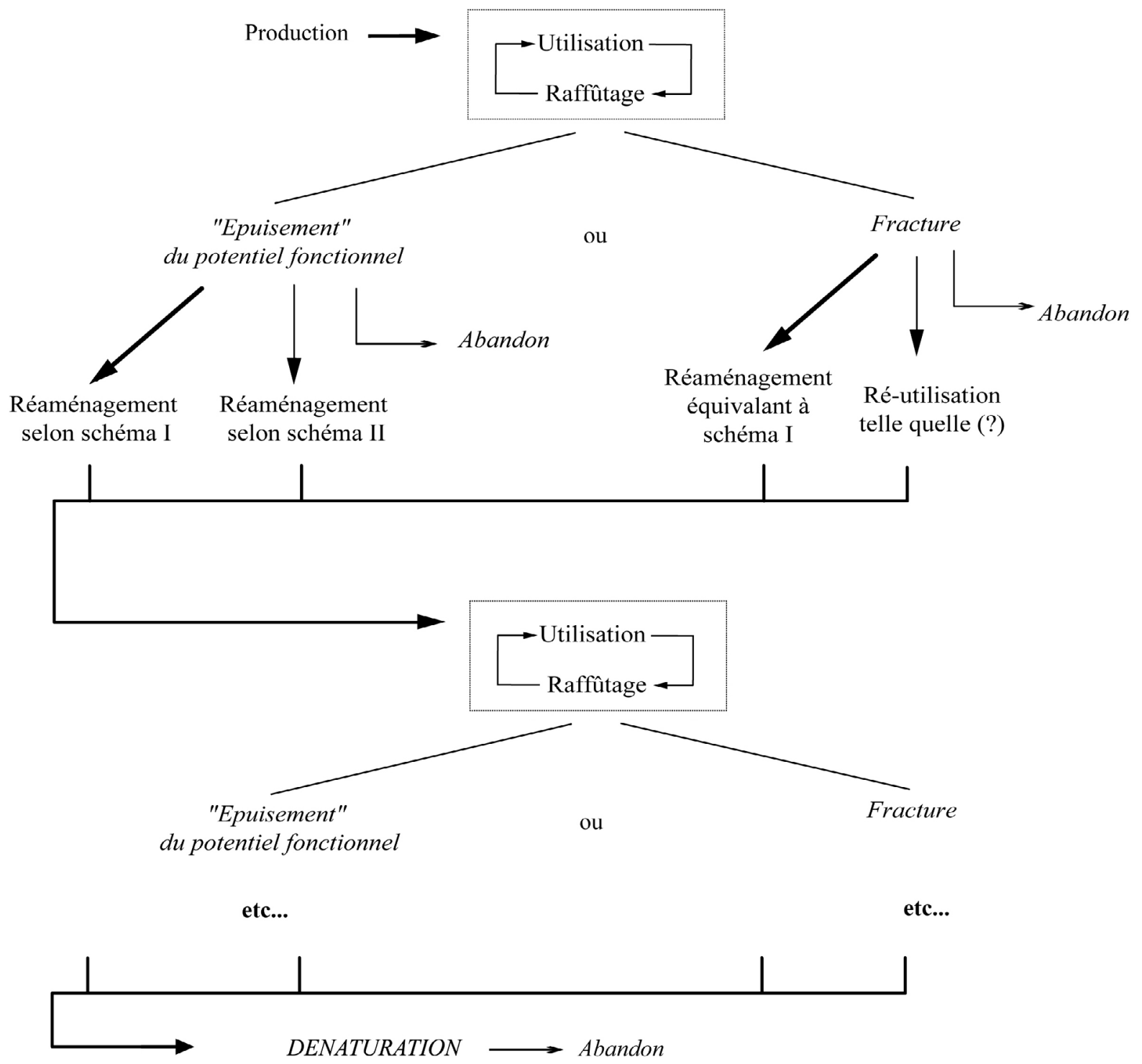

Figure 13. Représentation théorique des étapes par lesquelles passent les pièces façonnées unifacialement au cours de leur vie utile, depuis leur première utilisation jusqu’à leur dénaturation.

Figure 13. Theoretical diagram of stages through which unifacially shaped artefacts have gone along their uselife, since the first use to exhaustion. 


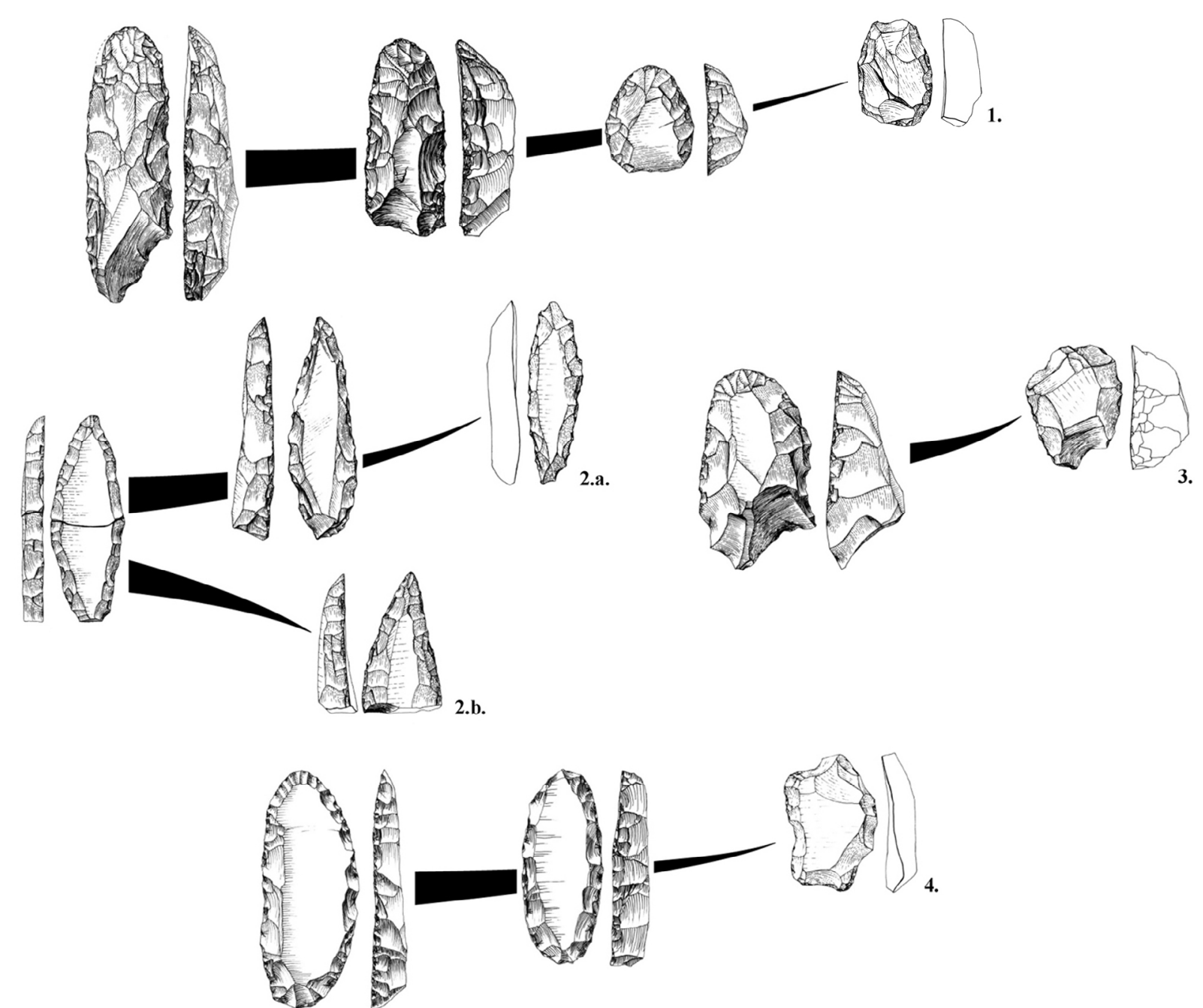

Figure 14. Exemples théoriques d'évolution des pièces façonnées unifacialement au cours de leur vie à partir de spécimens de la collection de GO-JA-01. 1, 2b, 3 et 4: Schéma de réaménagement I; 2.a : Schéma de réaménagement II.

Figure 14. Theoretical examples of transformation of unifacially shaped artefacts throughout their use-life (based on artefacts from GO-JA-01 rockshelter). 1, 2b, 3 and 4: Restructuration scheme I; 2a: Restructuration scheme II.

Tableau 3. Pièces façonnées unifacialement entières et dénaturées de GO-JA-01. Décompte et pourcentage des pièces pour chaque catégorie de section transversale.

Table 3. Complete and exhausted unifacially shaped artefacts from GO-JA-01. Count and percentage of pieces in each transversal section class.

\begin{tabular}{lccccc}
\hline Section transversale & Trapézoïdale & Semi-circulaire & Triangulaire & Indéterminée & Total \\
\hline Pièces entières & $44(40 \%)$ & $34(30,9 \%)$ & $32(29,1 \%)$ & - & 110 \\
Pièces dénaturées & $31(47 \%)$ & $19(28,8 \%)$ & $15(22,7 \%)$ & $1(1,5 \%)$ & 66 \\
\hline
\end{tabular}

Il y a donc une constance de la structure des pièces façonnées au cours du temps et la variabilité volumétrique observée ne saurait s’expliquer uniquement par la diachronie, dans la mesure où celle-ci existe quel que soit le degré de réaménagement de ces instruments. Il existe bien une diversité des intentions dès le début de la production.

Pour conclure, on peut se pencher sur les raisons de cette longue vie utile des pièces façonnées unifacialement du technocomplexe Itaparica. Cette économie des supports répondelle à des contraintes particulières du milieu extérieur des groupes qui les ont produits ? En ce qui concerne GO-JA-01, le grès silicifié, de très bonne qualité pour la taille, est abondant dans 
la région de Serranópolis, et notamment dans alentours directs du site. Un souci de rationnement de la matière première ne peut donc pas être invoqué pour expliquer l'intense exploitation des pièces façonnées unifacialement. Par ailleurs, d'après les données publiées sur d'autres sites du technocomplexe Itaparica, la longue vie utile de ces supports semble être la norme (Fogaça 2001; Bueno 2007; Lourdeau \& Pagli 2014). Les différences de contexte et de matières premières entre ces sites nous permettent d'écarter un lien entre cette gestion des supports aux cours du temps et des contraintes environnementales. Il semble bien que cette vie technique de longue durée, avec les changements qu'elle implique en termes de volume et de potentiels fonctionnels, soit une caractéristique imbriquée dans les autres propriétés structurelles des pièces façonnées unifacialement. Cela pourrait être mis en relation à une conception de l'espace et une structuration des territoires particuliers des groupes humains associés au technocomplexe Itaparica, au cours de ce premier moment d'occupation importante du centre et du nord-est du Brésil (Bueno 2011).

\section{Remerciements}

L'auteur tient à remercier Yan Axel Gómez Coutouly pour l'organisation de ce volume ainsi que Adrian Burke et un correcteur anonyme pour leurs commentaires ayant permis d'améliorer le texte d'origine.

\section{Références}

Boëda, E. 1997, Technogenèse de systèmes de production lithique au Paléolithique inférieur et moyen en Europe Occidentale et au Proche-Orient. Thèse d'Habilitation à diriger des recherches, Université Paris X-Nanterre, Nanterre, 149 p. (in French) (“Technogenesis of lithic production systems of Early and Middle Palaeolithic in Western Europe and Near-East”)

Boëda, E. 2001, Détermination des Unités Techno-Fonctionnelles de pièces bifaciales provenant de la couche acheuléenne C'3 base du site de Barbas I. In : Les industries à outils bifaciaux du Paléolithique moyen d'Europe Occidentale. Actes de la Table Ronde Internationale, Caen, 14-15 Octobre 1999 (Cliquet, D, Ed.), Études et Recherches Archéologiques de l'Université de Liège (ERAUL), Vol. 98. Université de Liège, Liège: p. 51-75. (in French) ("Determination of Techno-functional Units of bifacial pieces from Acheulean C'3 layer in the site of Barbas I")

Bueno, L. M. R. 2007, Variabilidade tecnológica nos sítios líticos da região do Lajeado, Médio Rio Tocantins, Suplementos da Revista do Museu de Arqueologia e Etnologia, Vol. 4. Museu de Arqueologia e Etnologia da Universidade de São Paulo, São Paulo, 215 p. (en portugais) ("Variabilité technologique dans les sites lithiques de la région de Lajeado, moyen cours du fleuve Tocantins") URL: http://www.revistas.usp.br/revmaesupl/article/view/113483

Bueno, L. 2011, L’occupation initiale du Brésil dans une perspective marcro-régionale : les cas de l'Amazonie, du Nordeste et du centre du Brésil. In: Peuplements et préhistoire en Amérique (Vialou, D, Ed.), Comité des Travaux Historiques et Scientifiques, Paris: p. 209-220. (in French) ("Initial settlement of Brazil from a macro-regional perspective: cases of Amazonia, Nordeste and Central Brazil”)

Bueno, L., Dias, A. D. \& Steele, J. 2013, The Late Pleistocene/Early Holocene archaeological record in Brazil: A geo-referenced database. Quaternary International, 301: 74-93. (en 
anglais) (“L’enregistrement archéologique du Pléistocène final/Holocène ancien au Brésil: une base de données géoréférencée”) doi:10.1016/j.quaint.2013.03.042

Dantas, M. D., Armesto, R. C. G. \& Adamy, A. 2008, Origem das paisagens. In: Geodiversidade do Brasil. Conhecer o passado para entender o presente e prever o futuro (Silva, C. R., Ed.), Companhia de Pesquisa de Recursos Minerais, Rio de Janeiro: p. 33-56. (en portugais) (“Origine des paysages”)

Fogaça, E. 1995, A Tradição Itaparica e as indústrias líticas pré-cerâmicas da Lapa do Boquete (MG-Brasil). Revista do Museu de Arqueologia e Etnologia de São Paulo, 5: 145-158. (en portugais) ("La tradition Itaparica et les industries lithiques précéramiques de Lapa do Boquete (Minas Gerais-Brésil)”) doi:10.11606/issn.24481750.revmae.1995.109233

Fogaça, E. 2001, Mãos para o pensamento. A variabilidade tecnológica de indústrias líticas de caçadores-coletores holocênicos a partir de um estudo de caso: as camadas VIII e VII da Lapa do Boquete (Minas Gerais, Brasil) - 12.000/10.500 B.P. Tese de Doutorado, Pontifícia Universidade Católica do Rio Grande do Sul, Porto Alegre, 452 p. (en portugais) (“Des mains pour la pensée. La variabilité technologique de l'industrie lithique de chasseurs-cueilleurs de l'Holocène à partir d'une étude de cas: les couches VIII et VII de Lapa do Boquete (Minas Gerais, Brésil) - 12.000/10.500 B.P.”)

Fogaça, E. 2003, Instrumentos líticos unifaciais da Transição Pleistoceno-Holoceno no Planalto Central do Brasil: individualidade e especificidade dos objetos técnicos. Canindé, 3: 9-35. (en portugais) (“Outils lithiques unifaciaux de la transition Pléistocène-Holocène dans le Plateau Central du Brésil: individualité et spécificité des objets techniques”)

Leroi-Gourhan, A. 1964, Le Geste et La Parole II - La Mémoire et Les Rythmes. Paris: Albin Michel, 285 p. (in French) (“Gesture and Speech II - Memory and Rythms”)

Lourdeau, A. 2010, Le technocomplexe Itaparica : Définition techno-fonctionnelle des industries à pièces façonnées unifacialement à une face plane dans le centre et le nordest du Brésil pendant la transition Pléistocène-Holocène et l'Holocène ancien. Thèse de Doctorat, Université Paris Ouest Nanterre La Défense, Nanterre, 477 p. (in French) ("The Itaparica technocomplex: techno-functional definition of industries with unifacially shaped artefacts with a plane face in Central and North-Eastern Brazil during the Pleistocene-Holocene transition and Early Holocene”)

URL: http://www.theses.fr/2010PA100190

Lourdeau, A. 2014, Les pièces façonnées unifacialement du techno-complexe Itaparica (centre et nord-est du Brésil) : concept et variabilité. In: Peuplement de l'Amérique du Sud: l'apport de la technologie lithique (Farías, M. \& Lourdeau, A., Eds.), Archéoéditions, Prigonrieux: p. 97-122. (in French) ("The unifacially shaped artefacts of the Itaparica technocomplex (Central and North-Eastern Brazil): concept and variability”)

Lourdeau, A. 2015, Lithic Technology and Prehistoric Settlement in Central and Northeast Brazil: Definition and Spatial Distribution of the Itaparica Technocomplex. PaleoAmerica, 1(1): 52-67. (en anglais) ("Technologie lithique et peuplement préhistorique du centre et du nord-est du Brésil: définition et distribution spatiale du Technocomplexe Itaparica”) doi:10.1179/2055556314Z.0000000005

Lourdeau, A. \& Pagli, M. 2014, Indústrias líticas pré-históricas na região da Serra da Capivara. In: Os biomas e as sociedades humanas na pré-história da região do Parque 
Nacional Serra da Capivara, Brasil (Pessis, A.-M., Martin, G. \& Guidon, N., Eds.), A \& A Comunicação, São Paulo: p. 551-635. (en portugais) ("Industries lithiques préhistoriques dans la region de la Serra da Capivara”)

Schmitz, P.I. 1987a, Prehistoric Hunters and Gatherers of Brazil. Journal of World Prehistory, 1(1): 53-126. (en anglais) (“Chasseurs-cueilleurs préhistoriques du Brésil”) doi:10.1007/BF00974817

Schmitz, P.I. 1987b, Caçadores antigos no sudoeste de Goiás, Brasil. Estudios Atacameños, 8: 17-37. (en portugais) (“Chasseurs anciens du sud-ouest de l’Etat de Goiás, Brésil”) doi:10.22199/S07181043.1987.0008.00004

Schmitz, P.I., Barbosa, A.S., Jacobus, A.L. \& Ribeiro, M.B. 1989, Arqueologia nos cerrados do Brasil Central. Serranópolis I. Pesquisas - Série Antropologia, Vol. 44. Instituto Anchietano de Pesquisas - UNISINOS, São Leopoldo, 208 p. (en portugais) (“Archéologie dans les cerrados du Brésil Central. Serranópolis I”)

Schmitz, P.I., Rosa, A.O. \& Bitencourt, A.L.V. 2004, Arqueologia nos cerrados do Brasil Central. Serranópolis III. Pesquisas - Série Antropologia, Vol. 60. Instituto Anchietano de Pesquisas - UNISINOS, São Leopoldo, 286 p. (en portugais) (“Archéologie dans les cerrados du Brésil Central. Serranópolis III”)

Soriano, S. 2000, Outillage bifacial et outillage sur éclat au Paléolithique ancien et moyen : coexistence et interaction. Thèse de Doctorat, Université Paris X - Nanterre, Nanterre, 459 p. (in French) ("Bifacial tools and flake tools during Early and Middle Palaeolithic: coexistence and interaction”) 


\title{
Life and death of a tool blank: Reconfiguration chaînes opératoires of unifacially shaped artefacts from the Itaparica technocomplex (Central Brazil)
}

\author{
Antoine Lourdeau ${ }^{1,2}$ \\ 1. Muséum National d'Histoire Naturelle, Département de Préhistoire, Musée de l'Homme, 17 place du \\ Trocadéro, 75116 Paris, France. \\ 2. Universidade Federal de Sergipe, Programa de Pós-graduação em Arqueologia, Laranjeiras, Brésil. \\ Email: antoine.lourdeau@mnhn.fr
}

\begin{abstract}
:
The Itaparica technocomplex encompasses most of the lithic productions in Central Brazil from the Pleistocene-Holocene transition and early Holocene. It is characterized by industries based on a techno-functional complementarity between tools made from unifacially shaped blanks and retouched flakes. The unifacially shaped artefacts, designated by different names in the literature (for example, limaces, plano-convex tools, unifaces), are historically the most emblematic remains of this technocomplex. Techno-functional analyses of these artefacts demonstrate that they did not correspond to a single tool, but rather to a blank for different simultaneous or successive tools. Many indicators also suggest that these blanks had a long useful live and passed through different technical phases. We present here a study of the chaînes opératoires related to the reconfiguration of the unifacially shaped artefacts of the Itaparica technocomplex during their use-life. To do so, we use the collection recovered during the excavation of the GO-JA-01 rockshelter, in Serranópolis (Goiás state), central Brazil. This important corpus of 377 pieces allows for a good perception of their technical variability. It comes from layers dated between 12,500 and 10,000 calibrated years BP.

Different stages of modification of the unifacially shaped artefacts can be observed. Resharpening is limited to restoring the functionality of an active part. It affects the sides as well as both extremities of the blank. It is common, also, to find these artefacts as fragments, due to transversal fractures. It is not always easy to determine if the fragments are the result of an accident during the use of the artefact or of a deliberate act of reconfiguration. Nonetheless, in various instances, cases of removal scars posterior to the fracture demonstrate that fragmentation was taken into account in the reconfiguration schemes of the unifacially shaped artefacts.

Strictly speaking, reconfigurations concern more important modifications than resharpening, affecting the artefact's volume and not only its periphery. They correspond to new steps of shaping, either partial or complete. Reconfigurations can be seen at the GO-JA-01 site thanks to detailed analyses to the diacritical schemes of production and by visible differences in artefact surfaces (e.g., double patinas). These modifications can follow one another until the blank is exhausted. In such cases, the artefact no longer possesses operational transformative parts.

By studying the indicators of modification throughout the use-life of the unifacially shaped artefacts from GO-JA-01, one can define two different reconfiguration schemes. In the predominant one, modifications touch mainly the distal and proximal ends, thus reducing the artefacts length. In a secondary scheme, on the contrary, width decreases due to reconfigurations in the sides. Those two schemes can also be found in the collection of artefacts from Lapa do Boquete site (Minas Gerais state).
\end{abstract}


Reconfigurations can have a significant impact on blank volume and form, but they cannot explain all the variability of these artefacts. It appears that there exists a consistency in the structure of the blanks over time and this calls for the existence of a diversity of intentions from the start of the production of the unifacially shaped artefacts of the Itaparica technocomplex.

Keywords: chaîne opératoire; lithic technology; tool use-life; unifacially shaped artefacts; Itaparica technocomplex; prehistory of Brazil 